Received: 31 May 2017

Accepted: 18 October 2017

Published online: 03 November 2017

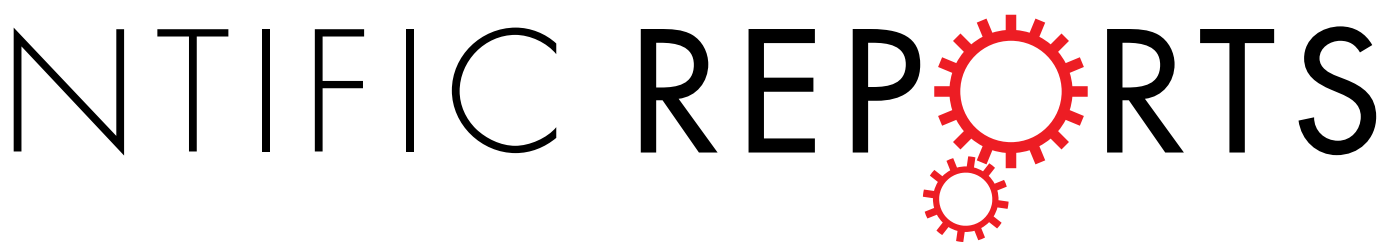

\title{
OPEN Biomineral Nano-Theranostic agent for Magnetic Resonance Image Guided, Augmented Radiofrequency Ablation of Liver Tumor
}

\author{
Anusha Ashokan ${ }^{1,3}$, Vijay Harish Somasundaram ${ }^{1}$, Genekehal Siddaramana Gowd ${ }^{1}$, \\ Ida M. Anna ${ }^{1}$, Giridharan L. Malarvizhi ${ }^{1}$, Badrinathan Sridharan ${ }^{1}$, Rupal B. Jobanputra ${ }^{1}$, \\ Reshmi Peethambaran ${ }^{2}$, A. K. K. Unni ${ }^{2}$, Shantikumar Nair ${ }^{1} \&$ Manzoor Koyakutty $^{1}$
}

Theranostic nanoparticles based on biocompatible mineral compositions can significantly improve the translational potential of image guided cancer nano-therapy. Here, we report development of a single-phase calcium phosphate biomineral nanoparticle ( $\mathrm{nCP}$ ) with dual-mode magnetic resonance contrast (T1-T2) together with radiofrequency (RF) mediated thermal response suitable for imageguided RF ablation of cancer. The nanoparticles (NP) are engineered to provide dual MR contrast by an optimized doping concentration (4.1 at\%) of paramagnetic ion, $\mathrm{Fe}^{3+}$, which also renders lossy dielectric character for $\mathrm{nCP}$ leading to thermal response under RF exposure. In vivo compatibility and dual-mode MR contrast are demonstrated in healthy rat models. MRI and T2 mapping suggest hepatobiliary clearance by $\sim 96$ hours. MRI guided intratumoral injection in subcutaneous rat glioma and orthotopic liver tumor models provide clear visualization of NP in MRI which also helps in quantifying NP distribution within tumor. Furthermore, by utilising RF mediated thermal response, NP treated tumor could be ablated using clinically approved RF ablation system $(10 \mathrm{~W}, 13.3 \mathrm{GHz})$. Real-time in vivo thermal imaging exhibits $119 \pm 10 \%$ increase in temperature change $(\Delta T)$ for NP treated orthotopic liver tumor $\left(\Delta T=51.5 \pm 2{ }^{\circ} \mathrm{C}\right)$, compared to untreated healthy liver control $\left(\Delta T=21.5 \pm 2{ }^{\circ} \mathrm{C}\right)$. In effect, we demonstrate a promising nano-biomineral theranostic agent for dual-mode MRI combined with radiofrequency ablation of solid tumors.

A theranostic agent is one which serves both diagnostic and therapeutic functions, a capability which will have obvious clinical advantages. With the advent of nanosystems for medical use, initial research focused separately on diagnostics and therapy. The former included quantum dots ${ }^{1-3}$ which faced challenges during translational phase due to toxicity concern $s^{4,5}$ and the latter included a variety of drug delivery systems ${ }^{6-11}$ where drugs were incorporated into biodegradable nanoparticles for the purpose of improving therapeutic efficacy. From the last one decade, there has been interest in combining both diagnostic and therapeutic functions into a single nanosystem, referred as nanotheranostics ${ }^{12-14}$. It would be helpful to define two types of nanotheranostic agents. In the first type, the drug and diagnostic agent are extrinsically loaded into a nanocarrier resulting in a multi-phase nanosystem ${ }^{12-15}$. In the second type, the single phase nanomaterial itself has both diagnostic and therapeutic functions ${ }^{16-18}$. The former can be termed as 'extrinsic' and the latter as 'intrinsic' nanotheranostic systems.

Most of the reported theranostic agents are extrinsic in nature, based on polymers ${ }^{19-22}$, dendrimers ${ }^{23-25}$, liposomes $^{26-29}$, or inorganic nanocarriers such as silica ${ }^{30-32}$ incorporating both diagnostic and therapeutic moieties.

${ }^{1}$ Center for Nanosciences and Molecular Medicine, Amrita University, Kochi, 682041, India. ${ }^{2}$ Central Lab Animal Facility, Amrita Institute of Medical Sciences, Amrita University, Kochi, 682041, India. ${ }^{3}$ Present address: Department of Biotechnology, Cochin University of Science and Technology, Kochi, 682022, India. Correspondence and requests for materials should be addressed to S.N. (email: shantinair@aims.amrita.edu) or M.K. (email: manzoork@aims. amrita.edu) 
Limitations of such extrinsic theranostic agents include complexity of incorporating multiple materials into a single nanosystem and challenges in obtaining high encapsulation efficacy. In contrast, intrinsic systems are relatively simple, single-phase materials with multi-functionality. Superparamagnetic iron oxide nanoparticles (SPIONs) is the only known intrinsic theranostic nanosystem reported so far for combining T2 weighted (dark) MR contrast together with magnetic hyperthermia property ${ }^{18,33}$. Although SPIONs is approved for T2 MR imaging, its application is limited due to prolonged in vivo clearance time ( $>30$ days $)^{34}$ and iron overload related ROS generation ${ }^{35,36}$.

Our group has been working in the area of biomineral nanocontrast agents since $2008^{37-39}$, especially on calcium phosphate/apatite nanoparticles, that has great potential for clinical translation due to their inherent compatibility as calcium phosphate is the major mineral component in human bone. Our earlier work focused on the development of multiple ions doped calcium phosphate nanoparticles (nCP) showing combined optical, magnetic and nuclear contrast properties ${ }^{37-39}$. Barth et al. used calcium phosphosilicate nanoparticles loaded with indocyanine green for targeted tumor imaging ${ }^{40}$ and photodynamic therapy ${ }^{41}$ in animal tumour models. Recently, the capability of calcium phosphate to dissolve at acidic $\mathrm{pH}$ was utilised by $\mathrm{Mi} \mathrm{P}$ et al. to develop $\mathrm{pH}$-activatable manganese doped calcium phosphate nanoparticle that showed enhanced magnetic contrast within acidic tumor microenvironment ${ }^{42}$. Calcium phosphate nanoparticles were also incorporated with a fluorophore along with drug, ceramide, for development of an extrinsic theranostic agent ${ }^{43}$. Our present work shows the potential of $\mathrm{nCP}$ to provide contrast and inherent therapeutic potential as an intrinsic theranostic agent.

In the present work, we optimized a unique intrinsic nanotheranostic property for nCP doped with 4.1 at $\%$ of $\mathrm{Fe}^{3+}$ (nCP:Fe) showing simultaneous dual-mode T1-T2 MR contrast and RF mediated thermal response suitable for MRI assisted RF ablation therapy of cancer. Dual-mode MRI helps in combining the merits of both T1 and T2 relaxivity values of the tissue ${ }^{44}$. T1 mode provides excellent soft tissue contrast together with anatomical details whereas T2 mode helps in the identification of lesions. So far, either paramagnetic gadolinium complexes ${ }^{45}$ or SPIONs ${ }^{46}$ were separately used for T1 or T2 imaging, respectively. Recently there were efforts to develop dual mode nanoconstructs by complexing ${ }^{47}$ or doping $\mathrm{Gd}^{3+}$ with $\mathrm{FeO}^{48}$. Instead of using heavy-metals such as $\mathrm{Gd}^{3+}$, a potential nephrotoxic element, dual mode MR contrast using low-dose $\mathrm{Fe}^{3+}$ doped (1.5-4.1 at \%) biomineral calcium phosphate (nCP) may be a better alternative. Most importantly, $\mathrm{Fe}^{3+}$ doped $\mathrm{nCP}$ showed unique dielectric-loss characteristics under RF exposure, resulting in increase in temperature on exposure to low-power $(<10 \mathrm{~W})$ radio-waves. Radiofrequency ablation (RFA) is clinically utilised for minimally invasive ablation of solid tumors including hepatocellular carcinoma (HCC) and renal cancer. A major drawback of current RFA is the inferior thermal conductance of tissue, limiting the size of the lesion that can be treated to $<5 \mathrm{~cm}^{49}$. In clinics, this problem is addressed by repeated repositioning of RF electrodes in order to cover the entire disease area or by intratumoral injection of ionic solutions such as saline to improve RFA efficiency ${ }^{50}$. There are also a few reports where nanostructures such as nano-gold ${ }^{51}$, carbon nanotubes ${ }^{52}$ and graphene ${ }^{53}$ were utilized for enhancing the efficacy of RFA. However, all these materials are non-biodegradable and may pose long-term toxicity effects. Furthermore, there are no theranostic nanoparticles reported so far for minimally invasive, MR image guided RFA. Although, Merkle et al. investigated the feasibility of using $\mathrm{FeO}$ nanoparticles for MR guided RFA of liver, there was no significant increase in coagulation diameter observed in vivo ${ }^{33}$. To the best of our knowledge, this is the first report on single-phase bio-mineral intrinsic theranostic nanoparticle providing dual mode MR contrast and RFA response.

\section{Results and Discussion}

Synthesis and Characterization of nCP:Fe. Our main objective was to optimize a therapeutic functionality in Fe doped nCP together with dual mode T1 and T2 MR contrast property. In our previous reports on doped nCP for multimodal imaging ${ }^{37-39}$, we used Gadolinium as the doping agent for MR contrast. In the present work, instead of $\mathrm{Gd}^{3+}$, which may cause nephrogenic systemic fibrosis in patients with chronic kidney disease $\mathrm{s}^{54}$, we used a more biocompatible $\mathrm{Fe}^{3+}$ to generate paramagnetic property in $\mathrm{nCP}$. We hypothesize that at appropriate doping concentrations, $\mathrm{Fe}^{3+}$ can also induce lossy dielectric character for $\mathrm{nCP}$ at radio-frequency range that would make the material suitable for RFA.

Figure $1 \mathrm{~A}$ shows the schematic lattice arrangement of $\mathrm{nCP}: \mathrm{Fe}$ where $\mathrm{Fe}^{3+}$ possibly substitute the cationic position of $\mathrm{Ca}^{2+}$ or occupy interstitial space. An obvious change in body-color from white (undoped nCP) to brown was observed after doping with $\mathrm{Fe}^{3+}$ (Fig. 1B). TEM images (Fig. 1C) showed well dispersed spherical nanoparticles of average size $\sim 90 \mathrm{~nm}$ which was confirmed from DLS analysis (Fig. 1D) that gave a size distribution of $125 \pm 60 \mathrm{~nm}$. X-ray diffraction (Supplementary Figure S1A) and electron diffraction pattern (Inset Fig. 1C) indicated amorphous nature of nCP:Fe. During the reaction between calcium and phosphate precursors for $\mathrm{nCP}: \mathrm{Fe}$ synthesis, it was critical to maintain the $\mathrm{pH}$ at $\sim 9$ for obtaining spherical nanoparticles, as neutral or acidic $\mathrm{pH}$ (pH 5-7) resulted in formation of rod shaped particles as reported earlier by our group ${ }^{38}$. In order to prevent the aggregation and crystal growth after synthesis, we surface capped the nanoparticles with citrate ions. Adsorption of citrate ions on calcium phosphate facets contributes to controlled growth of nanoparticles ${ }^{55}$. This interaction was also reflected in the relatively high negative zeta potential, $-15 \mathrm{mV}$ of capped nCP:Fe (Fig. 1E). Inductively coupled plasma (ICP) analysis showed an average doping efficiency of $\sim 40 \%$ (Table 1 ). Further, we also investigated the washing out of $\mathrm{Fe}^{3+}$ ions from $\mathrm{nCP}$ :Fe, for which the final supernatant obtained after fourth wash of sample was analyzed by ICP. $\mathrm{Fe}^{3+}$ level in supernatant was below $1 \mathrm{ppm}$, indicating no significant leakage of $\mathrm{Fe}^{3+}$ from nCP:Fe. Magnetic property analyzed by VSM showed linear increase in magnetization with external field, indicating paramagnetic behavior compared to the diamagnetic property of undoped nCP (Fig. 1F). With increase in doping concentration from 0.5 to 6 at $\%$ an increase in paramagnetic behavior (Fig. $1 \mathrm{~F}$ ) and magnetic susceptibility (Supplementary Figure S1B) was observed. There are reports on the paramagnetic ${ }^{56}$ or superparamagnetic ${ }^{57}$ behavior of $\mathrm{Fe}^{2+}$ or $\mathrm{Fe}^{3+}$ incorporated hydroxyapatite depending on the ionic state of doped $\mathrm{Fe}$ and phase of the host matrix. The paramagnetic nature of nCP:Fe in the present case may be conferred to the presence of lone unpaired electron in the $3 \mathrm{~d}$ orbital of $\mathrm{Fe}^{3+}$. 

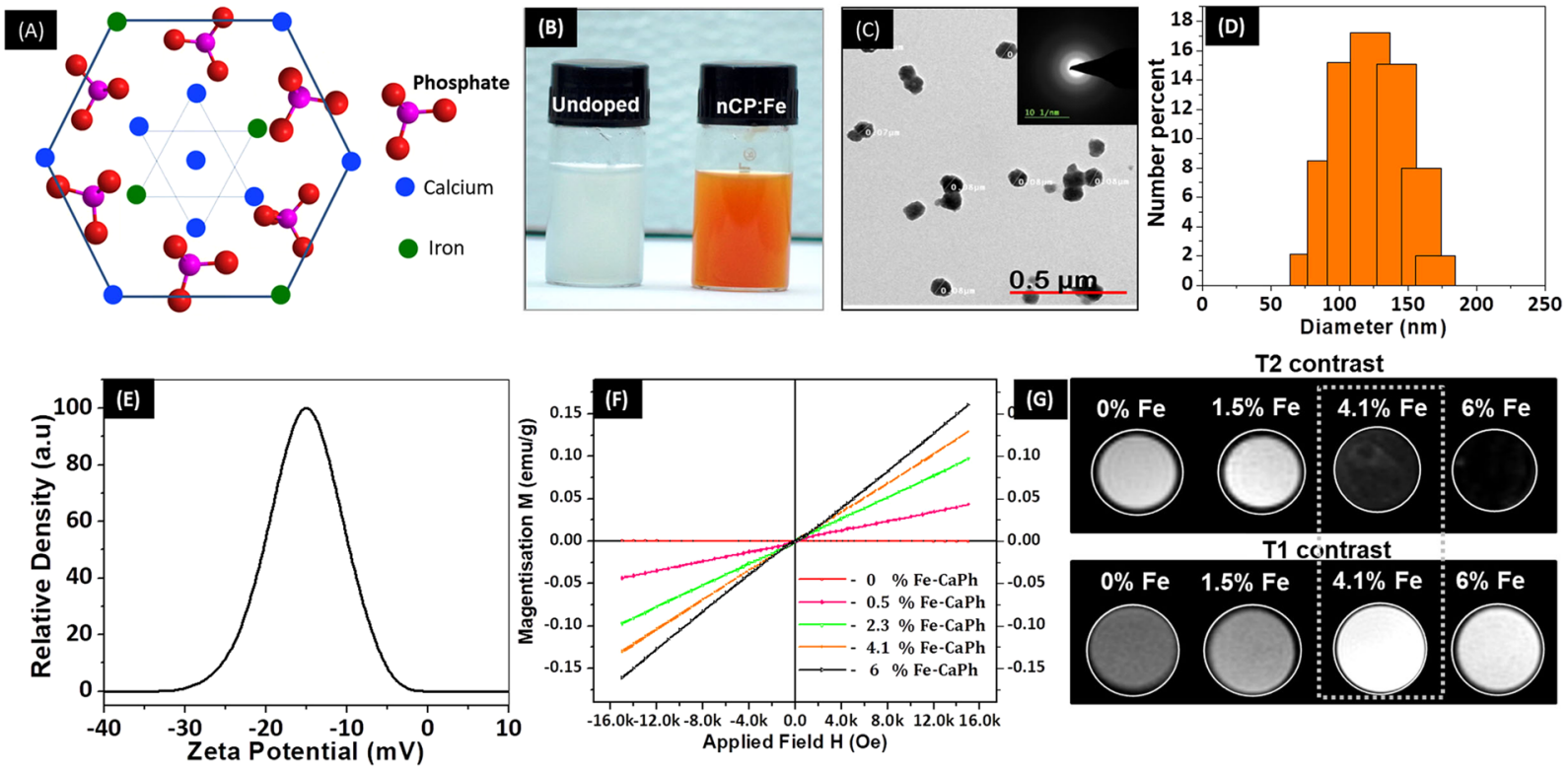

Figure 1. Characterization of nCP:Fe. (A) Schematic of nCP:Fe where a few $\mathrm{Ca}^{2+}$ atoms are replaced by $\mathrm{Fe}^{3+}$ (B) Photograph of undoped nCP (left) and nCP:Fe (right) solution at concentration of $10 \mathrm{mg} / \mathrm{mL}$. (C) TEM image of nCP:Fe, Inset: ED pattern (D) DLS showing size distribution (E) Zeta potential data (F) VSM data of different batches of nCP:Fe doped with varying concentration of $\mathrm{Fe}^{3+}$. (G) T2 and T1 weighted MR contrast of $1.25 \mathrm{mg} / \mathrm{mL}$ of $\mathrm{nCP}: \mathrm{Fe}$ doped with varying concentration of $\mathrm{Fe}^{3+}$, dispersed in agar phantom. $4.1 \%$ Fe doped nCP showed an optimized dual T1-T2 contrast that is marked in white dotted box.

\begin{tabular}{|l|l|}
\hline$\% \mathrm{Fe}^{3+}$ added during synthesis & $\% \mathrm{Fe}^{3+}$ doped within $\mathrm{nCP}$ (ICP data) \\
\hline 1 & 0.5 \\
\hline 5 & 2.3 \\
\hline 10 & 4.1 \\
\hline 15 & 6 \\
\hline
\end{tabular}

Table 1. Actual $\mathrm{Fe}^{3+}$ doping \% (calculated from ICP data) compared to $\% \mathrm{Fe}^{3+}$ added during synthesis.

Dual mode T1-T2 weighted MRI of nCP:Fe. Next, we investigated the T1 and T2 weighted magnetic contrast property of nCP:Fe. Contrast property of paramagnetic nanoparticles is highly sensitive to the concentration of doped ions ${ }^{58}$. The $\mathrm{T} 1$ effect (bright contrast) dominates at lower concentrations of paramagnetic impurity due to spin-lattice interactions between the paramagnetic ions and protons whereas T2 effect (dark contrast) dominates at higher concentrations due to spin-spin interactions between high concentration of paramagnetic ions ${ }^{37}$. Utilizing this property of paramagnetic ions, in the present work, we optimized $\mathrm{Fe}^{3+}$ doping concentration to obtain an enhanced $\mathrm{T} 1$ and $\mathrm{T} 2$ relaxation rates that would lead to both bright contrast in $\mathrm{T} 1$ mode and dark contrast in the T2 mode of MRI. Figure $1 \mathrm{G}$ shows that an increase in $\mathrm{Fe}^{3+}$ dopant concentration from 1.5 to 6 at\% resulted in an enhancement of T2 contrast intensity whereas T1 contrast was maximum at 4.1 at $\%$ which reduced with higher doping. This suggests that spin-lattice relaxation rate dominated till 4.1 at $\%$ doping after which spin-spin relaxation rate was higher leading to T2 contrast. Therefore we optimized the doping concentration at 4.1 at $\%$ to obtain an effective T1 and T2 relaxation rates suitable for dual mode T1-T2 contrast in MRI. Mapping studies carried out to estimate the T1 and T2 relaxivity values of 4.1 at\% Fe doped nCP gave $r 1=0.75 \mathrm{mM}^{-1} \mathrm{~s}^{-1}$ and $r 2=29.6 \mathrm{mM}^{-1} \mathrm{~s}^{-1}$ (Supplementary Figure S1C, D). Although these values are relatively lower than that of typical contrast agents (Gd-DOTA: $r 1=2.8 \mathrm{mM}^{-1} \mathrm{~s}^{-1}$, SPIO: $r 2=213 \mathrm{mM}^{-1} \mathrm{~s}^{-1}$ at $7 \mathrm{~T}$ ), this is optimum for the development of a dual mode T1-T2 contrast agent for magnetic contrast assisted intratumoral injection for MR guided therapeutics in clinics. Thus, we selected 4.1 at $\% \mathrm{Fe}^{3+}$ doped $\mathrm{nCP}$ as optimum for dual mode T1-T2 contrast imaging in MRI and this sample is hereafter referred as $\mathrm{nCP}: \mathrm{Fe}$.

In vitro compatibity of nCP:Fe. Prior to in vivo studies, we tested the compatibility of nCP:Fe towards primary human peripheral blood derived mononuclear cells (PBMC) and RBC. Blood compatibility assessment is a prerequisite for preclinical testing of nanoparticle derived medicines and medical devices. PBMC viability analysis showed excellent compatibility up to $500 \mu \mathrm{g} / \mathrm{mL}$ after 48 hours of treatment (Supplementary Figure S2A). Hemolysis assay (Supplementary Figure S2B) also showed that nCP:Fe does not disturb the RBC membrane integrity up to a tested concentration of $250 \mu \mathrm{g} / \mathrm{mL}$ which was further confirmed by SEM imaging (Supplementary Figure S2B Inset). It was also important to check the ROS generation capability of nCP:Fe as there are a few 
reports that discuss the possible stress mechanism activated by SPIONs which is ROS mediated ${ }^{36}$. DCFH-DA mediated ROS analysis in N1S1 cells showed that nCP:Fe did not induce any additional ROS (11.3 $\pm 1.5 \%)$ compared to PBS control ( $12.5 \pm 0.5 \%$ ) (Supplementary Figure S2C, D) whereas $\sim 100 \%$ of hydrogen peroxide treated cells produced significant ROS (Supplementary Figure S2E). The compatibility of nCP:Fe to the blood cells and low ROS generation may be positively considered for using $\mathrm{nCP}: \mathrm{Fe}$ as an alternate MR contrast agent.

In vivo dual mode (T1-T2) MR Imaging in healthy rat and tumor models. Reports on in vivo dual mode MRI used either two separate contrast agents injected one after the other ${ }^{59}$ or an extrinsic nanoparticle incorporating two different magnetic impurities (Gd and SPIO) for $\mathrm{T} 1$ and $\mathrm{T} 2$ contrast $^{47,48}$. To evaluate the in vivo dual mode T1-T2 contrast property of nCP:Fe, $10 \mathrm{mg} / \mathrm{kg}$ sample was intravenously injected to healthy rats. After 30 minutes, an enhancement of both T1 and T2 contrast was observed especially in the liver and heart region as shown in white dotted box in Fig. 2A,B. T2 mapping analysis showed that reduction in T2 contrast in liver region was also associated with lowering of T2 relaxation time from $40 \pm 1 \mathrm{mS}$ to $25 \pm 1 \mathrm{mS}$. Axial T2 weighted images of liver sections before (Fig. 2C) and after (Fig. 2D) nCP:Fe injection clearly showed enhancement in T2 contrast intensity. T2 mapping data (Supplementary Figure S3) obtained from selected ROI (white circles in Fig. 2C,D) reflected the enhanced relaxivity changes in the liver after nCP:Fe injection.

We also evaluated the biodistribution and clearance time of nCP:Fe after intravenous injection. T2 weighted whole body coronal (Fig. 2E) and axial (Fig. 2F) MRI showed an increase in T2 contrast in liver (shown in white dotted box) within the first one hour which gradually reduced to initial (before injection) contrast by 96 hours. This variation was also reflected in T2 relaxation values that drastically reduced in the first hour after injection (from $40 \pm 1 \mathrm{mS}$ to $25 \pm 1 \mathrm{mS}$ ) and then gradually increased to initial value $40 \mathrm{mS}$ by $\sim 96$ hours (Fig. $2 \mathrm{G}$ ). This clearly indicates initial nanoparticle uptake by mononuclear phagocyte system within the liver and subsequent clearance over a period of 96 hours. This was confirmed by tissue-ICP analysis after 1 hour of injection which showed an increase in $\mathrm{Fe}^{3+}$ content in all organs as well as plasma with significantly higher concentration in the heart, plasma and liver (Fig. 2H). We believe that the increase in Fe content in plasma is not due to the degraded nanoparticles because our in vitro studies on nCP:Fe treated RAW 264.7 macrophages showed less degradation even after 12 hours of intracellular localization (Supplementary Figure S4). These results suggest that the nanoparticles circulated in the body and accumulated in the liver within 1 hour and slowly cleared by 96 hrs. We compared the clearance time of nCP:Fe vs SPIONS (in healthy rats) after intravenous injection using T2 values of liver and found that nCP:Fe cleared within 4-5 days whereas SPIONS was not cleared upto 15 days (Supplementary Figure S5). Our previous observation on ICG and $\mathrm{Gd}^{3+}$ co-doped calcium phosphate nanoparticles indicated hepatic clearance of $\mathrm{nCP}^{37}$. Compared to the changes observed in the T2 relaxation values of liver, post nCP:Fe injection, we have not observed any significant contrast changes in kidneys. Therefore we assume that the nCP:Fe nanoparticles were cleared through the hepatobiliary route similar to ICG and Gd doped nCP.

Considering the potential clinical application of intratumoral nCP:Fe injection for MRI assisted radiofrequency ablation therapy, we tested its ability to provide dual mode contrast in the tumor region after direct injection into the subcutaneous tumor mass. $10 \mathrm{mg} / \mathrm{kg}$ of sample was injected to the tumor, grown in Wistar rat model. Excellent T2 (Fig. 3A,B) and T1 contrast (Fig. 3C,D) was observed from the sample injected region as shown in two different slices. The contrast change also correlated with the reduction of relaxation times, T2 from $48 \pm 2$ to $18 \pm 1 \mathrm{mS}$ and $\mathrm{T} 1$ from $2096 \pm 3$ to $340 \pm 2 \mathrm{mS}$. Interestingly, imaging after 18 hours showed an increase in distribution of nanoparticles from $0.046 \pm 0.001 \mathrm{~cm}^{3}, 10$ minutes after injection (Fig. 3E,F), to $0.056 \pm 0.005 \mathrm{~cm}^{3}$, 18 hours after injection (Fig. $3 \mathrm{G}, \mathrm{H}$ ). This also led to changes in contrast properties. Dark contrast in T2 mode (Fig. 3E) turned bright (Fig. 3G), clearly indicating dilution of paramagnetic nanoparticles due to the increased diffusion into larger tumor area. This was also noted in T1 imaging (Fig. 3F,H) and mapping, as T1 relaxation time increased from $340 \pm 2 \mathrm{mS}$ (at the time of injection) to $840 \pm 4 \mathrm{mS}$ (after 18 hours). By normalizing the tissue contribution to T1 value and approximating the tumor as a homogenous tissue, we have calculated the concentration of nanoparticles distributed over the tumor region, from the T1/T2 values compared to the injected dose ( $3 \mathrm{mg})$. We found that $\sim 2.5 \mathrm{mg}$ was the distributed dose after 18 hours in a tumor volume of $0.056 \pm 0.005 \mathrm{~cm}^{3}$. Thus, we demonstrated excellent T1 and T2 contrast property of nCP:Fe injected intratumoral regions and showed its capability to diffuse throughout the tumor region up to $1-1.8 \mathrm{~cm}$, which could be quantified by T1/T2 mapping. The capacity of nanoparticles to distribute throughout tumor and the ability to view and quantify the dose present at tumor site in a specified time is critical in implementing theranostic dosimetry.

In vitro radiofrequency response of $\mathrm{nCP}: \mathrm{Fe}$. After the optimization of MR contrast properties and tissue distribution, we investigated if the doping of Fe within $\mathrm{nCP}$ changed its dielectric loss property which could be utilized for its application in RFA. Variation in dielectric loss was tested using impedance analyzer for a frequency range of $0-700 \mathrm{kHz}$. We found that in the frequency range applied for RF ablation $(350-550 \mathrm{kHz})$, the dielectric loss, $\tan \delta$, increased from 2.4 (undoped $\mathrm{nCP}$ ) to 3.54 (4.1\% Fe doped $\mathrm{nCP}$ ) at $450 \mathrm{KHz}$ (Fig. 4A). This clearly indicated enhanced dielectric loss character due to $\mathrm{Fe}^{3+}$ doping in the bioceramic, $\mathrm{nCP}$. Earlier reports on the dielectric loss properties of calcium phosphosilicate glass doped with iron oxide $\left(\mathrm{Fe}_{2} \mathrm{O}_{3}\right)$ was attributed to the increased ionic conductance of iron in a deformed calcium phosphosilicate structure ${ }^{60}$. We expect similar features in $\mathrm{nCP}: \mathrm{Fe}$, where doped $\mathrm{Fe}$ may have increased the ionic conductance of $\mathrm{nCP}: \mathrm{Fe}$ leading to increase in dielectric loss. Further, we compared the RF mediated heating of undoped $\mathrm{nCP}$ and $\mathrm{nCP}: \mathrm{Fe}$ at varying $\mathrm{NP}$ concentration at $100 \mathrm{~W}$ RF for 1 minute exposure using a non-invasive RF machine (Schematic: Fig. 4B). In the concentration range of $50-500 \mu \mathrm{g} / \mathrm{mL}$, significant increase in solution temperature leading to temperature rise, $\Delta \mathrm{T}, 22 \pm 1.5^{\circ} \mathrm{C}$ (maximum temperature $50^{\circ} \mathrm{C}$ ) was observed for $\mathrm{nCP}$ : Fe compared to $\Delta \mathrm{T}<10^{\circ} \mathrm{C}$ (maximum temperature $38^{\circ} \mathrm{C}$ ) for undoped nCP (Fig. 4C). Rise in temperature up to $50^{\circ} \mathrm{C}$ will be sufficient to initiate an ablative response in tumor cells ${ }^{61}$. 

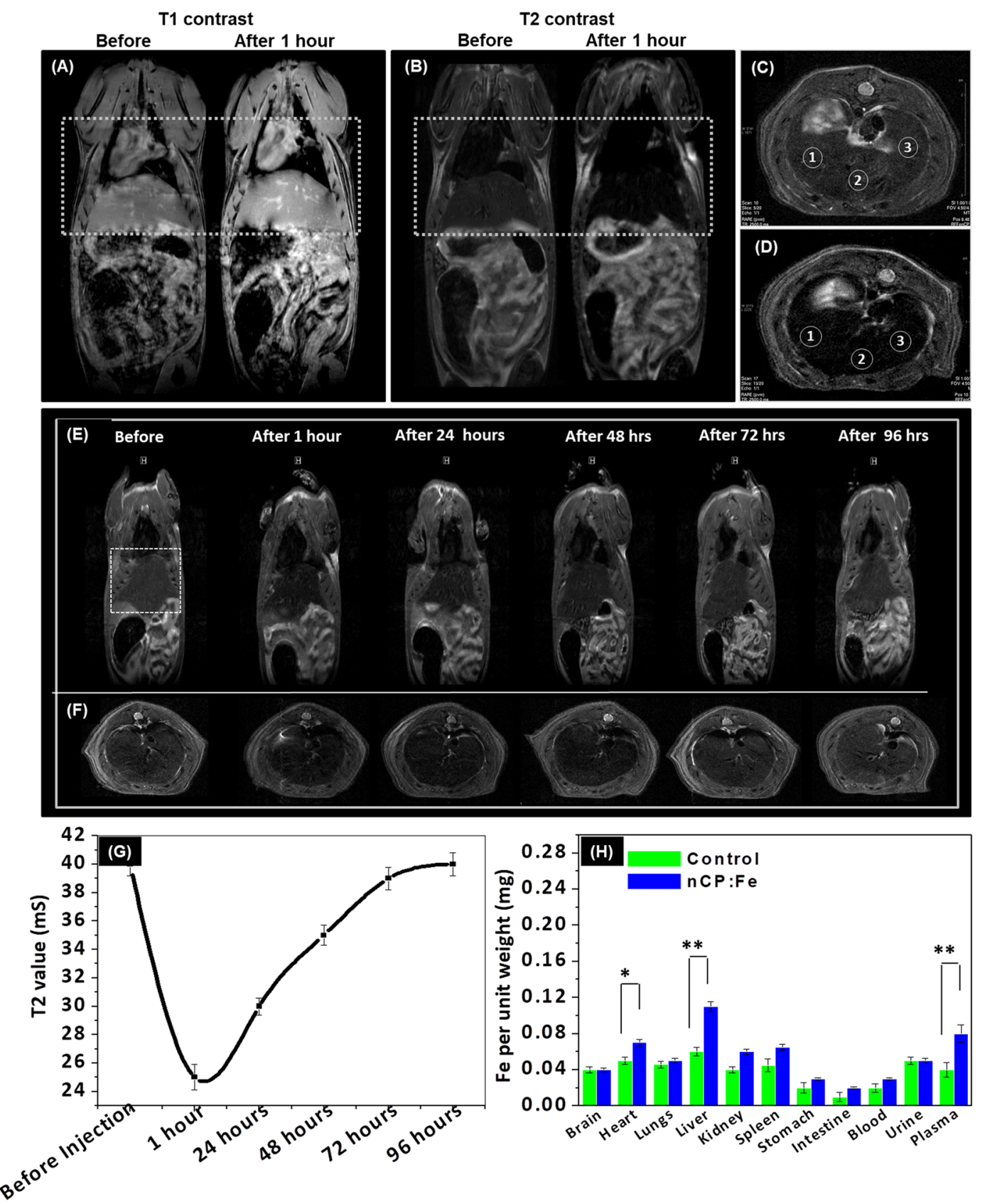

Figure 2. In vivo dual T1-T2 contrast and biodistribution of $\mathrm{nCP}$ :Fe. In vivo (A) T1 weighted and (B) T2 weighted MRI (coronal section) of Wistar rat before and after nCP:Fe injection. Enhancement of both T1 and T2 contrast can be observed after sample injection in liver and heart region (white dotted box) (C) Axial liver section before sample injection (D) Axial liver section 30 minutes after sample injection (E) T2 weighted MRI of wistar rat over a period of 96 hours after intravenous injection of $\mathrm{nCP}: \mathrm{Fe}(\mathrm{F})$ Corresponding axial liver sections over a period of 96 hours (G) Variation in T2 time of liver over a period of 96 hours after nCP:Fe injection (H) Fe content in different organs, 1 hour after sample injection, compared to PBS control, estimated by ICP analysis.

To evaluate RF mediated cell death under in vitro conditions, N1S1 hepatoma cells were treated with both nCP and nCP:Fe for 4 hours followed by non-invasive RF treatment (100 W, 5 minutes exposure). Figure $4 \mathrm{D}$ shows significant reduction in viability for nCP:Fe treated cells compared to that of undoped nCP. $300 \mu \mathrm{g} / \mathrm{mL}$ of $\mathrm{nCP}: \mathrm{Fe}$ reduced the cell viability to $5 \pm 0.5 \%$ whereas $31 \pm 2 \%$ of cells remained live when treated with same concentration of undoped nCP. Optical images of control cells showed round morphology 48 hours after RF treatment (Fig. 4E) whereas nCP:Fe treated cells lost membrane integrity and was found disintegrated (Fig. 4F). Next, we investigated the RF response in goat liver phantom tissue. Goat liver was collected from 3 different animals and divided into two groups (nCP:Fe group and PBS control group) each containing 2 phantoms from each animal. We injected samples at a concentration of $10 \mathrm{mg} / \mathrm{kg}$ to the tissue at a fixed depth of $1 \mathrm{~cm}$ and applied RF $(10 \mathrm{~W}$ 


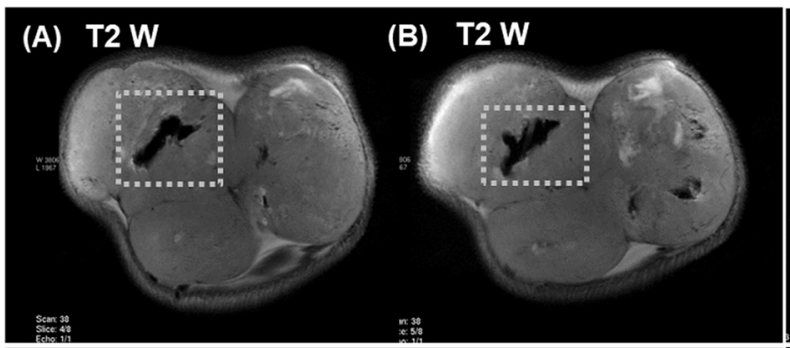

10 min after injection
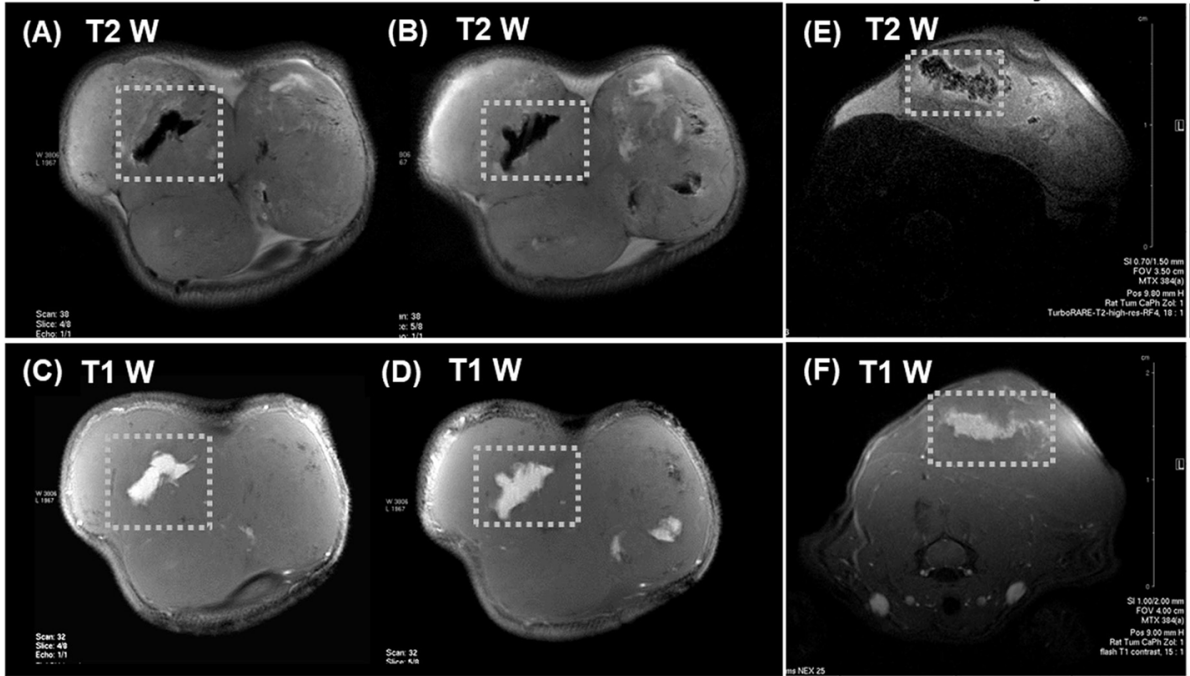

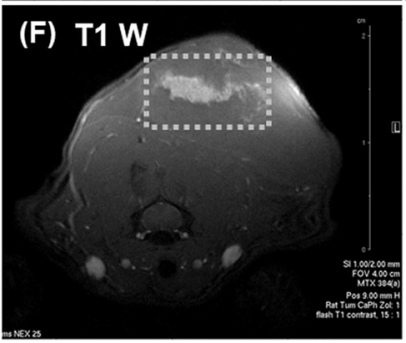

18 hrs after injection
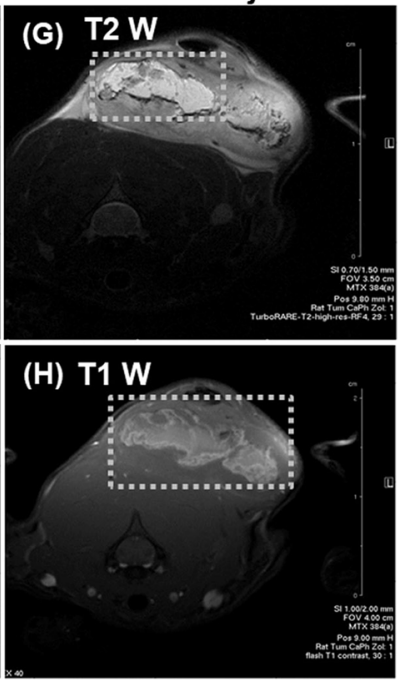

Figure 3. Dual T1-T2 contrast variation after intra-tumoral injection. (A,B) T2 weighted and (C,D) T1 weighted MR image (coronal section) of subcutaneous tumor after intratumoral nCP:Fe injection. (E) T2 weighted and (F) T1 weighted MRI of subcutaneous tumor (axial sections) 10 mins after intratumoral injection of nCP:Fe. (G) T2 weighted and (H) T1 weighted MRI of subcutaneous tumor (axial sections) 18 hours after intratumoral injection of nCP:Fe. Sample injected regions are shown in white dotted boxes.
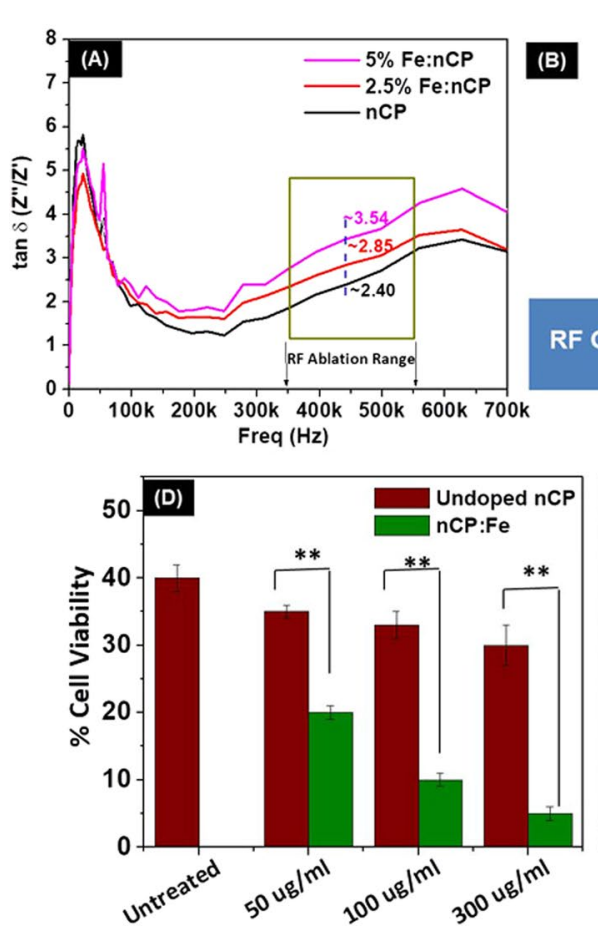

RF cabin

(B) Radiofrequency waves
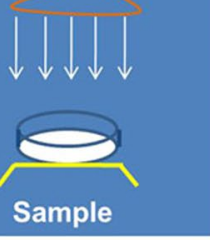

RF Generator

(E)

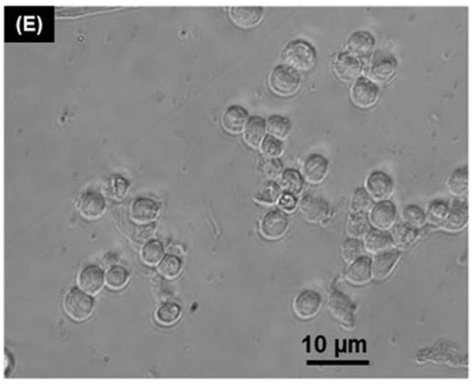

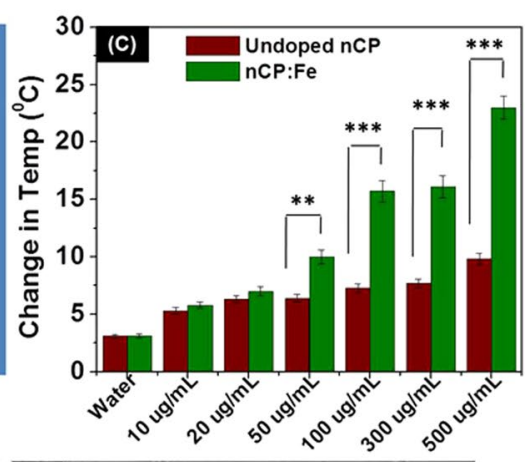

(F)

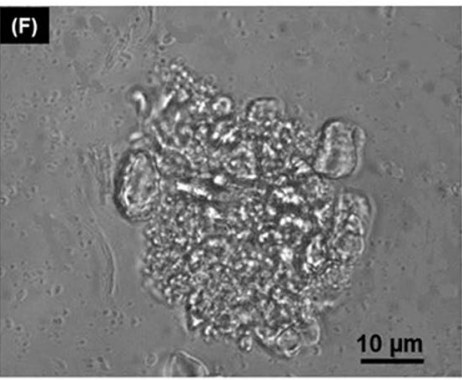

Figure 4. Radiofrequency response of $\mathrm{nCP}$ :Fe and its effect on cell viability. (A) Impedance spectroscopy analysis showing variation of $\tan \delta$ value of $\mathrm{nCP}$ :Fe with frequency (B) Schematic of non-invasive RF system (C) Change in temperature obtained by RF exposure of different concentration of nanoparticles at $100 \mathrm{~W}$ for 1 minute. (D) Viability of nCP:Fe/nCP treated N1S1 hepatoma cells, 4 hours after RF treatment (100 W power was applied for 5 minutes). Viability test was done 48 hours after RF exposure. Optical microscope images of (E) untreated (F) nCP:Fe treated cells, 48 hours after RF exposure.

for 1 minute) using clinical RFA system. $87 \%$ increase ( $8 \pm 1 \mathrm{~mm}$ for PBS control group to $14 \pm 2 \mathrm{~mm}$ for $\mathrm{nCP}$ :Fe group) in the diameter of RF ablated necrotic area was noted for nCP:Fe injected tissue phantom (Fig. 5B) compared to the PBS injected control tissue (Fig. 5A). This temperature difference between the ablated tissues was 


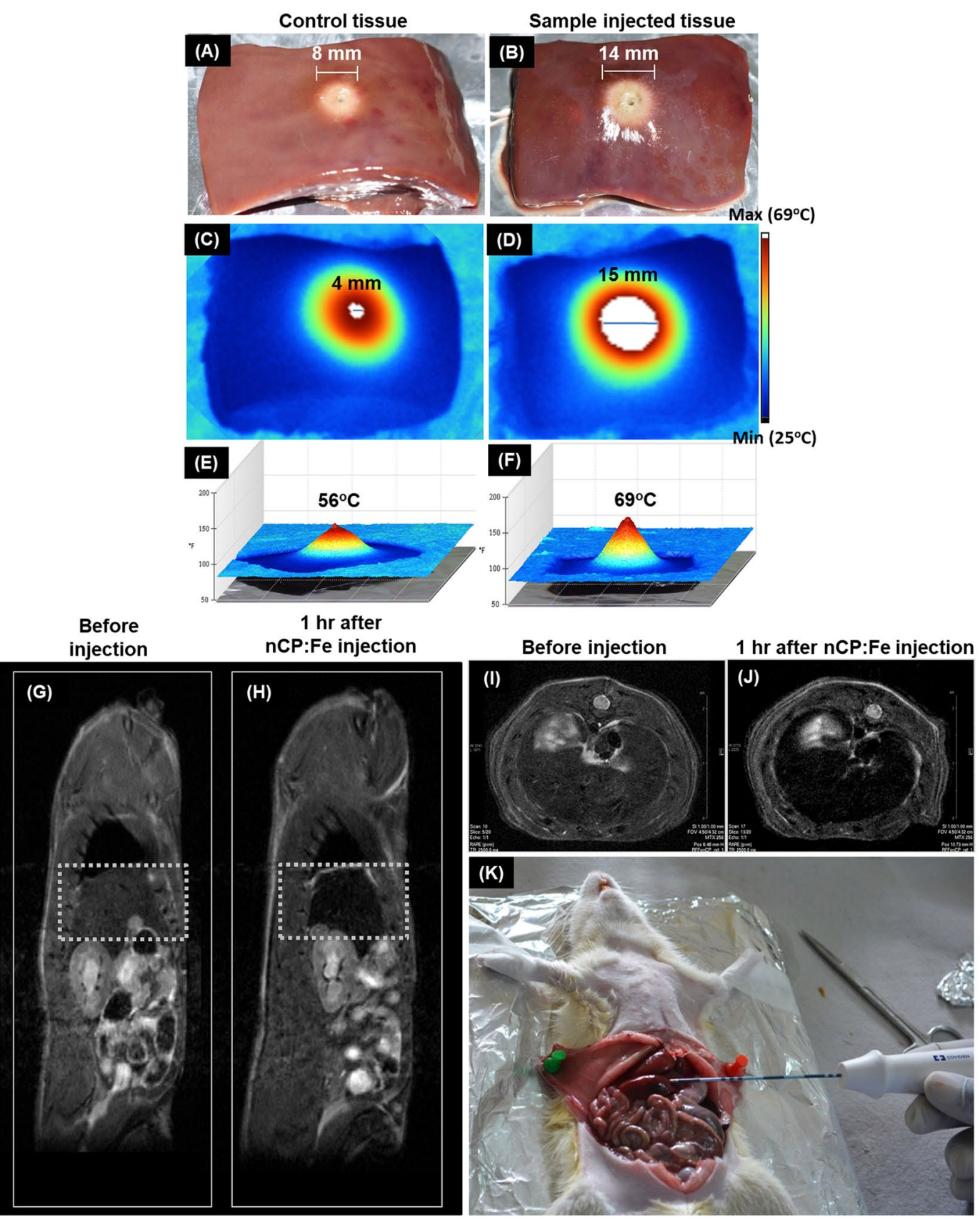

Figure 5. Radiofrequency ablation of phantom goat liver and healthy rat liver tissue after nCP:Fe injection. (A) Control (PBS injected) goat liver phantom tissue after RFA (ablation diameter $8 \pm 1 \mathrm{~mm}$ ) (B) nCP:Fe injected phantom tissue after RFA showing enhanced ablation diameter of $14 \pm 2 \mathrm{~mm}(\mathbf{C}-\mathbf{F})$ Corresponding IR images and 3D temperature plots of the ablated tissue (C,E) Control tissue after RFA gave peak temperature of $56 \pm 2{ }^{\circ} \mathrm{C}$, hot-spot diameter of $4 \pm 1 \mathrm{~mm}(\mathbf{D}, \mathbf{F})$ Sample injected tissue after RFA gave peak temperature of $69 \pm 2.5^{\circ} \mathrm{C}$, hot-spot diameter of $15 \pm 1 \mathrm{~mm}$. T2 weighted MRI (coronal section) of rat (G) before and $(\mathbf{H})$ 1 hour after nCP:Fe injection. Liver is shown in dotted box. T2 weighted MRI of liver (axial section) (I) before and (J) 1 hour after sample injection (K) RF ablation of rat liver being carried out using clinically used cooled tip RF probe.

imaged in real-time using a hand-held IR thermal imaging camera. The images (Fig. 5D,F) clearly showed that nCP:Fe injected tissue heated to a peak temperature of $69 \pm 2.5^{\circ} \mathrm{C}$ whereas the control tissue was heated to only $56 \pm 2{ }^{\circ} \mathrm{C}$ (Fig. 5C,E). The hot-spot (maximum temperature region) diameter for nCP:Fe was $15 \pm 1 \mathrm{~mm}$ (Fig. 5D) versus $4 \pm 1 \mathrm{~mm}$ (Fig. 5C) for PBS control. On varying the angle between the sample and IR camera, there is a possibility for change in temperature recorded at different angles. Therefore we calculated the error that can happen by measuring a constant temperature (dish of distilled water heated to $35^{\circ} \mathrm{C}$ ) at different angles $\left(0^{\circ}\right.$ to $\left.90^{\circ}\right)$ and found that the maximum deviation is $\pm 0.45^{\circ} \mathrm{C}$ (Supplementary Figure S6). This is well within the error value reported $\left( \pm 1.5^{\circ} \mathrm{C}\right)$ in the mean temperature value in IR images. Here we used a plane surface (dish of distilled 

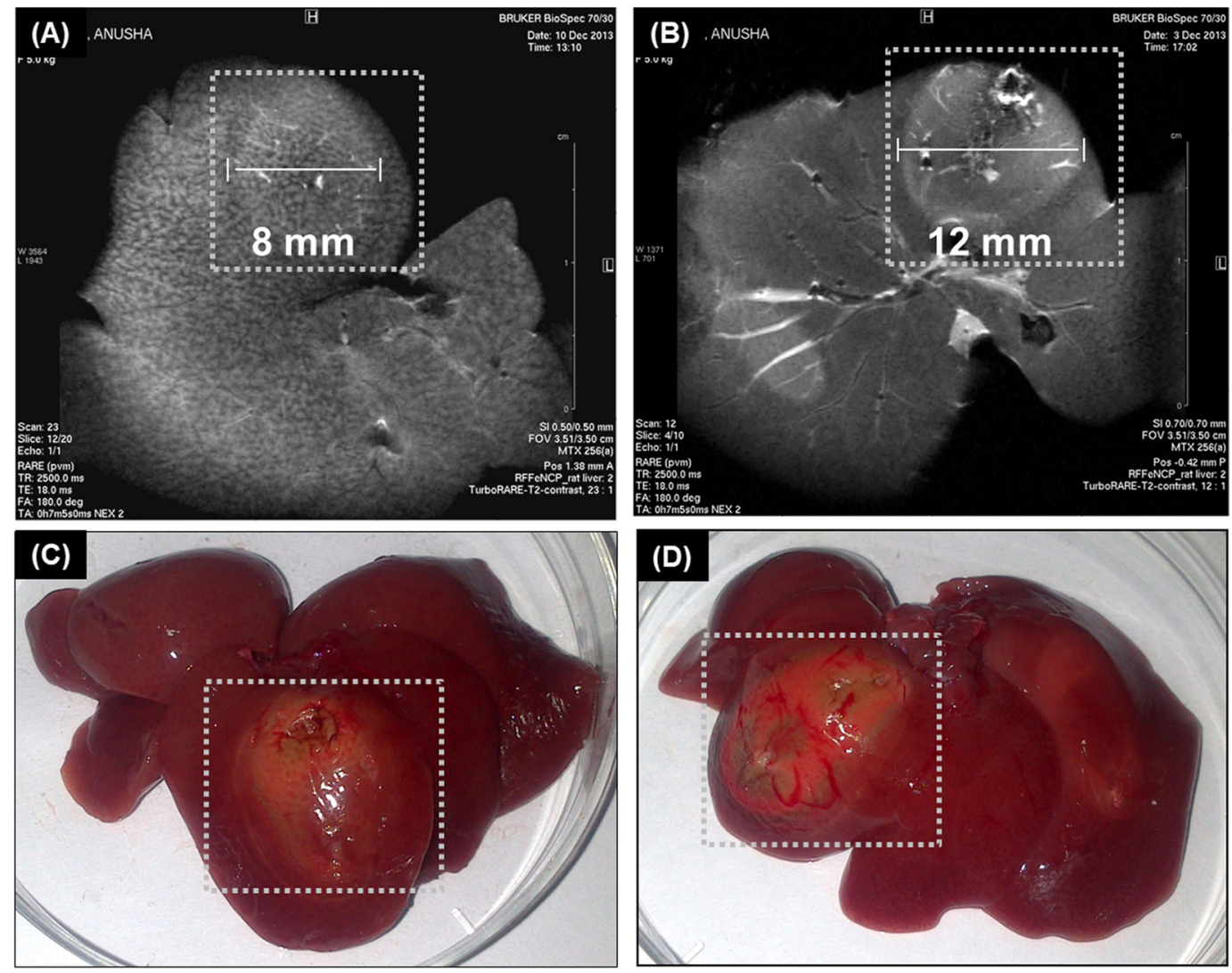

\section{(E)}
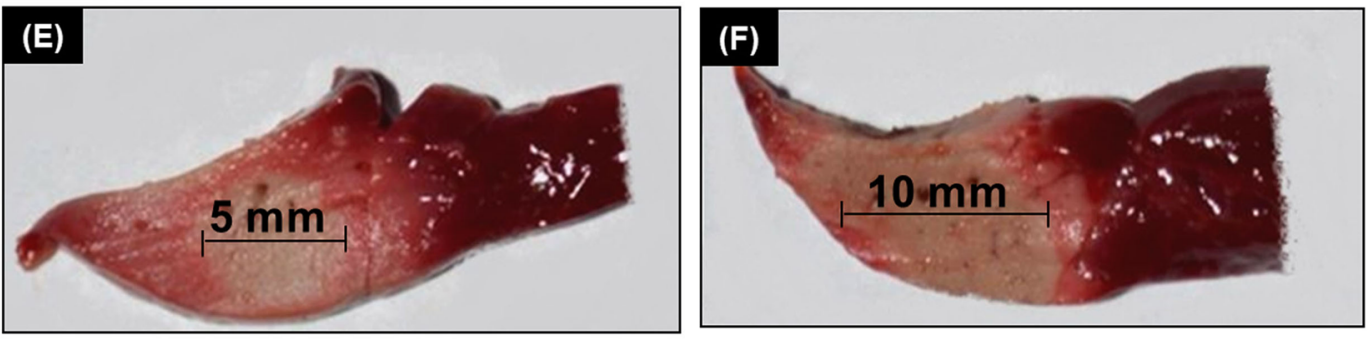

Figure 6. Response to RFA evaluated by MRI and viability staining. T2 weighted MRI of liver (axial section) after RF exposure (A) liver from control animal injected with PBS (B) liver from animal injected with nCP:Fe. Region of necrosis is shown in white dotted box (C) Photograph of liver from control animal and (D) liver from sample injected animal. Region of necrosis is shown in white dotted box. Viability staining by TTC of liver sections from (E) control animal (F) animal injected with nCP:Fe.

water) to calculate error, whereas in in vivo conditions the surfaces are curved that can increase the error value. Therefore, whenever possible, the angle of the IR camera should be maintained perpendicular to the curved surface under analysis. Thus the phantom tissue data clearly showed enhanced ablative effects of nCP:Fe compared to PBS. This ablative effect combined with dual mode (T1-T2) MR contrast properties reveals the potential of using nCP:Fe for MRI guided RFA.

In vivo MRI assisted RFA of healthy liver and orthotopic liver tumor. To evaluate the capability of $\mathrm{nCP}: \mathrm{Fe}$ to provide MR image assisted application of RFA in vivo, $10 \mathrm{mg} / \mathrm{kg} \mathrm{nCP}: \mathrm{Fe}$ was intravenously injected to Wistar rat. Two groups (PBS control group and nCP:Fe group), each containing 3 animals were taken. Sagittal whole body (Fig. $5 \mathrm{G}, \mathrm{H}$ ) and axial liver MRI (Fig. $5 \mathrm{I}, \mathrm{J}$ ) showed clear T2 contrast enhancement in the liver, one hour after nCP:Fe injection. RFA was then carried out under anesthesia at $10 \mathrm{~W}$ power for 1 minute as shown in Fig. 5K. After RFA, MRI of axial liver section showed contrast enhancement in the necrotic region for the PBS control (Fig. 6A) and nCP:Fe injected (Fig. 6B) animal. The diameter of RFA induced necrosis was measured as $8 \pm 1 \mathrm{~mm}$ for control (Fig. $6 \mathrm{~A}, \mathrm{C}$ ) and $12 \pm 1 \mathrm{~mm}$ (Fig. $6 \mathrm{~B}, \mathrm{D}$ ) for sample injected animal. MR images (Fig. 6B) clearly demarcated the necrosed region in nanoparticle injected animal compared to the control (Fig. 6A), indicating the enhanced T2 effect contributed by the NP. This is clinically relevant because in MR guided saline perfused RFA, the saline injection reduces MR contrast which made it difficult to assess the extent of necrosis ${ }^{62}$. In the present case, $\mathrm{nCP}$ :Fe mediated contrast enhancement helped to better evaluate the actual therapeutic response by way of measuring the extent of coagulation necrosis during and after RFA. RF mediated necrosis was also 

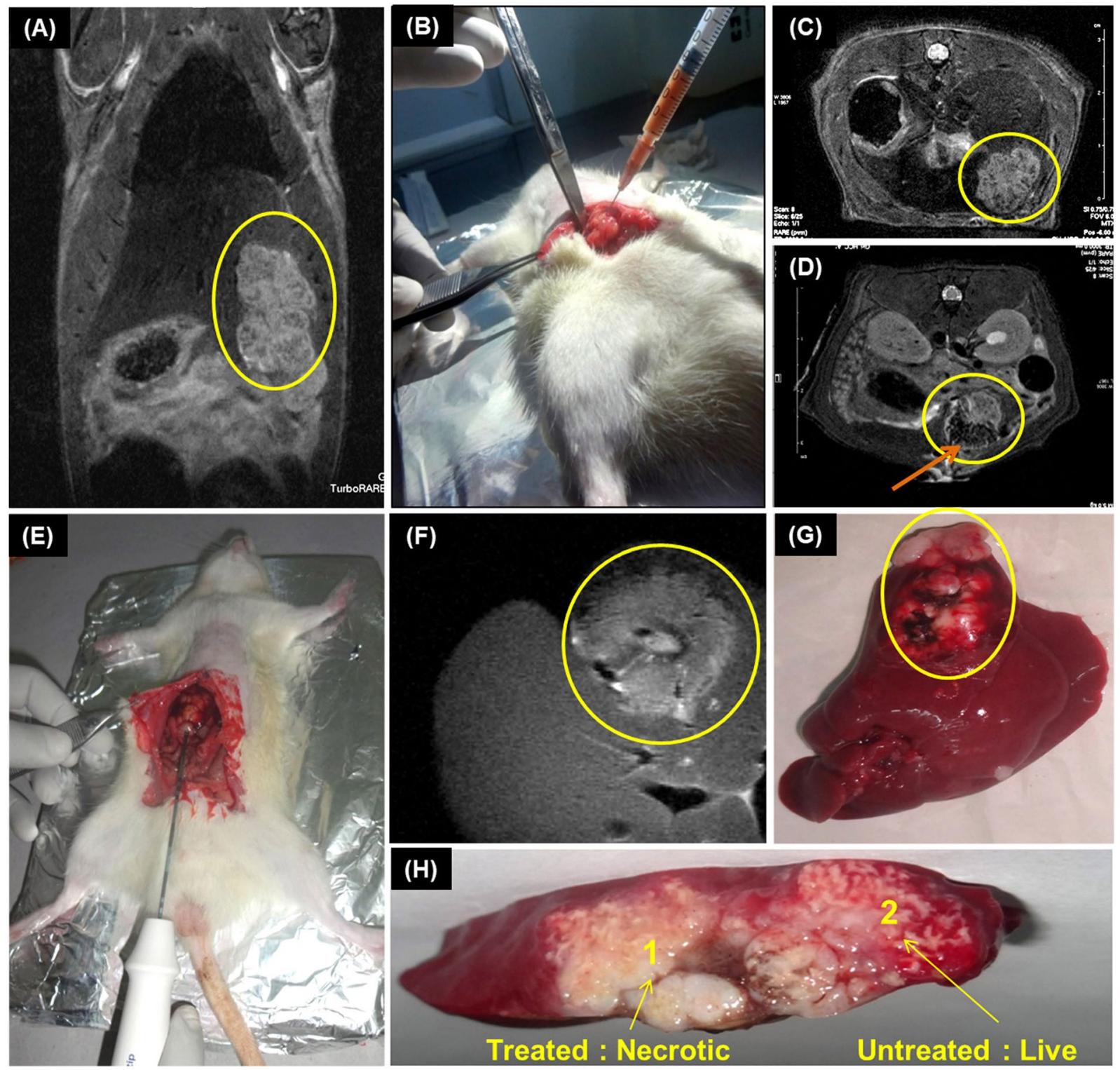

Figure 7. MRI assisted RFA in orthotopic liver tumor model. (A) Coronal T2 weighted MRI of tumor model showing liver tumor in encircled region (B) Intratumoral injection of $300 \mu \mathrm{L}$ of $10 \mathrm{mg} / \mathrm{ml} \mathrm{nCP}$ :Fe. T2 weighted axial MRI (C) before and (D) after intratumoral sample injection. Tumor region marked within yellow circle. The dark region within the tumor (arrow mark) is due to T2 contrast of nCP:Fe. (E) RFA of the liver tumor. Probe was inserted to tumor (at intersection of injected and non-injected area), $10 \mathrm{~W}$ power was applied for 1 minute (F) Axial MRI of the liver showing the bright ablated region (G) Photograph of the rat liver showing ablated tumor within circle $(\mathbf{H})$ TTC stained liver section showing sample treated necrotic tumor (unstained: Area 1) and untreated control tumor showing viability (stained pink: Area 2).

confirmed by tissue-viability staining using triphenyltetrazolium chloride (TTC) that stains viable tissue pink and leave the necrosed tissue unstained. TTC staining showed an increased area of complete necrosis (unstained area of $\sim 10 \mathrm{~mm}$ ) in the $\mathrm{nCP}$ :Fe injected liver section (Fig. $6 \mathrm{~F}$ ) compared to control (unstained area of $\sim 5 \mathrm{~mm}$ ) (Fig. 6E). Histological analysis of the liver tissue confirmed the RF mediated necrosis in nanoparticle treated liver (Arrow mark, Supplementary Figure S7B), which is showed in comparison to healthy liver region without RF treatment (Supplementary Figure S7A).

Next, we investigated the efficacy of nCP:Fe for MR assisted RFA in orthotopic liver tumor (N1S1) model. Considering intratumoral injection of RF enhancers in clinics, we directly injected nCP:Fe into tumor lobe which was followed by MR assisted RFA. For proof of concept, we carried out the experiment in 3 orthotopic rat liver tumor models. The tumor was well demarcated in the coronal whole body (Fig. 7A) and axial liver (Fig. 7C) MRI and marked in yellow circles. In order to keep a control region within the same tumor, we have considered two different tumor lobes of same tumor for the study. nCP:Fe was injected to the lower tumor lobe (Fig. 7B) resulting in excellent dark contrast in T2 weighted axial liver MRI section (arrow in Fig. 7D). RFA was applied 
at the interface of sample injected and non-injected lobe (Fig. 7E) using a clinical RF probe at a low dose of $10 \mathrm{~W}$ for 1 minute. The necrosis due to ablation was clearly seen in the MRI (Fig. 7F). Treated liver was resected out (Fig. 7G) and viability staining by TTC was done to examine the extent of ablation. Figure 7H clearly showed specific areas of necrosis (no stain area, Mark-1) in nCP:Fe injected tumor lobe compared to the untreated control lobe (pink stain area, Mark-2). This experiment also showed that nanoparticles remained well within the injected region of tumor and effect of RFA was confined to the same region. This justifies our proposal to administer nCP:Fe as intra-tumoral formulation rather than intravenous injection where healthy liver cells may also take up nanoparticles and undergo non-specific ablation.

To confirm the differential ablative effects in the nanoparticle treated tumor versus untreated liver region, we have done separate experiments where IR thermal imaging was employed to capture the real-time temperature increase during the application of RFA. Two groups (1. Orthotopic liver tumor rats 2. Healthy rat as control), each containing 3 animals were used for this study. In the orthotopic rat liver tumor animals, NP were injected into the tumor region and RFA was applied. Healthy liver without nanoparticle injection was used as control. Figure 8A, $\mathrm{B}$ shows visual image and IR thermal image, respectively of sample being injected intratumorally to animals just before the RFA. With the application of RF, $10 \mathrm{~W}$ for 1 minute (Fig. $8 \mathrm{C}$ ), temperature at the RF treatment point raised to $81.3 \pm 1.5^{\circ} \mathrm{C}$ (starting temp: $29.8 \pm 2{ }^{\circ} \mathrm{C}$ ) in nCP:Fe treated tumor (IR image: Fig. $8 \mathrm{D}$ ) whereas in the control healthy liver (Fig. 8E), maximum temperature obtained was only $51.3 \pm 2{ }^{\circ} \mathrm{C}$ (IR image: Fig. $8 \mathrm{~F}$ ) as plotted in Fig. 8G. Effectively, the nCP:Fe nanoparticles caused $119 \pm 10 \%$ increase in $\Delta \mathrm{T}$ of tumor region $\left(\Delta \mathrm{T}_{\text {avg }}=51.5^{\circ} \mathrm{C}\right)$ compared to the control $\left(\Delta \mathrm{T}_{\text {avg }}=21.5^{\circ} \mathrm{C}\right)$. Thus we demonstrated the capability of nCP:Fe as a theranostic agent for MR assisted RFA of liver tumor.

\section{Conclusion}

In summary, we have developed a novel theranostic biomineral nanoparticle using iron doped calcium phosphate ( $\mathrm{nCP}: \mathrm{Fe}$ ) that could provide dual mode (T1-T2) magnetic resonance contrast enhancement together with thermal response suitable for ablation of solid tumors on exposure to clinically approved radiofrequency range and power. Magnetic characterization showed increase in paramagnetic susceptibility with $\mathrm{Fe}^{3+}$ doping. Optimum concentration of $\mathrm{Fe}^{3+}$ required for dual mode T1-T2 contrast was optimized at 4.1 at \% doping with relaxivity values, $r 2=29.6 \mathrm{mM}^{-1} \mathrm{~s}^{-1}$ and $r 1=0.75 \mathrm{mM}^{-1} \mathrm{~s}^{-1}$. In vivo dual mode MR imaging capability of nanoparticles was demonstrated in rat models. Bio-distribution analysis using whole body T2 imaging and mapping showed maximum liver accumulation by $\sim 1$ hour after sample injection followed by complete clearance by $\sim 96$ hours. Radiofrequency impedance measurement showed an increase in dielectric loss factor $(\tan \delta)$ due to doping with $\mathrm{Fe}^{3+}$ and the nanoparticles exhibited excellent RF mediated thermal response $\left(100 \mathrm{~W}, 1\right.$ minute) with $\Delta \mathrm{T}=22^{\circ} \mathrm{C}$ in water medium. In vitro cell response to RF after $\mathrm{nCP}$ :Fe treatment showed $26 \%$ increased cell death compared to undoped nCP treated cells. MRI assisted RFA, demonstrated in healthy rat liver, led to enhanced RF mediated necrosis in nCP:Fe treated tissue (necrosis diameter $12 \pm 1 \mathrm{~mm}$ ) compared to PBS control (necrosis diameter $8 \pm 1 \mathrm{~mm}$ ). In orthotopic liver tumor model, $\mathrm{nCP}:$ Fe injected tumor region showed $119 \pm 10 \%$ increase in $\Delta \mathrm{T}$ (Final temperature: $81.3 \pm 1.5^{\circ} \mathrm{C}$ ) compared to the untreated healthy liver region (Final temperature: $51.3 \pm 2{ }^{\circ} \mathrm{C}$ ). Thus, we demonstrated the synthesis and in vivo theranostic application of a novel biomineral nanoparticle, nCP:Fe, for dual-mode MR image assisted radiofrequency ablation of liver tumor. Compared to any other engineered nanoparticles, calcium phosphate, being a major mineral component of our body, possesses excellent translational potential.

\section{Methods}

Synthesis of nCP:Fe. In a typical reaction procedure, $20 \mathrm{~mL}$ of $0.5 \mathrm{M}$ calcium chloride $\left(\mathrm{CaCl}_{2}, \operatorname{Sigma}\right.$, USA) was mixed with $20 \mathrm{~mL}$ of $0.2 \mathrm{M}$ trisodium citrate $\left(\mathrm{Na}_{3} \mathrm{C}_{6} \mathrm{H}_{5} \mathrm{O}_{7}\right.$, Fisher Scientific, India) and $0.1 \mathrm{M} \mathrm{FeCl}_{3}\left(\mathrm{Sigma}_{\text {, }}\right.$ USA). Volume of $0.1 \mathrm{M} \mathrm{FeCl}_{3}$ added was varied as per the required percentage of doping. $5 \mathrm{~mL}$ of $0.3 \mathrm{M}$ diammonium hydrogen phosphate $\left(\left(\mathrm{NH}_{4}\right)_{2} \mathrm{HPO}_{4}\right.$, S.D Fine Chemicals, India) mixed with $0.2 \mathrm{~mL}$ of $3 \mathrm{~N}$ ammonium hydroxide $\left(\mathrm{NH}_{4} \mathrm{OH}\right.$, Fisher Scientific, India) was added drop wise to the above mixture of $\mathrm{CaCl}_{2}, \mathrm{Na}_{3} \mathrm{C}_{6} \mathrm{H}_{5} \mathrm{O}_{7}$ and $\mathrm{FeCl}_{3}$ under constant stirring to obtain nCP:Fe. The precipitate was washed 4 times in hot distilled water by centrifugation at $8500 \mathrm{rpm}$ for 15 minutes and redispersed in PBS for further studies.

Characterization of nCP:Fe. XRD of nCP:Fe was analyzed using PANanalytical X Pert-pro system fitted with $\mathrm{Cu}-\mathrm{K} \alpha$ source. Particle size analysis was done using transmission electron microscope (Tecnai, G2, FEI) and dynamic light scattering (Nano ZS, ZetasizerNanoseries, Malvern). Zeta potential was measured using Nano ZS, ZetasizerNanoseries, Malvern. $\mathrm{Fe}^{3+}$ content within nCP:Fe was analyzed by Inductively Coupled Plasma-Atomic Emission Spectroscopy (ICP-AES Thermo Electron IRIS INTREPID II XSP DUO). $10 \mathrm{mg}$ of nCP:Fe was dissolved in 5\% nitric acid for ICP-AES analysis. Magnetic property of nCP:Fe was analyzed in vibration sample magnetometer (ADE EV7 1.5 T). T1 and T2 weighted magnetic contrast imaging was done in $7 \mathrm{~T}$ animal MRI station (BrukerBioSpec, USA). $1.25 \mathrm{mg} / \mathrm{mL}$ of nCP:Fe with varying concentration of doped $\mathrm{Fe}^{3+}$ was dispersed in $1 \%$ agar phantoms and MRI was carried out. T2 and T1 weighted MRI parameters were TR $=2500 \mathrm{mS}, \mathrm{TE}=33 \mathrm{mS}$, $\mathrm{FA}=180^{\circ}$ and $\mathrm{TR}=8.5 \mathrm{mS}, \mathrm{TE}=2.2 \mathrm{mS}, \mathrm{FA}=15^{\circ}$ respectively. Relaxivity values, $r 1$ and $r 2$, were calculated from T1 and T2 mapping data for different concentrations of nCP:Fe. Dielectric loss of nCP:Fe was calculated from frequency response impedance measurements in frequency response analyzer (Nova Impedance Spectroscopy). For impedance measurement of $\mathrm{nCP}: \mathrm{Fe}$, powder sample was made into pellet and sandwiched between Indium Tin Oxide coated glass slides. To make proper contact silver paste was applied on both sides of pellet and using clips connected to the frequency response analyzer. 

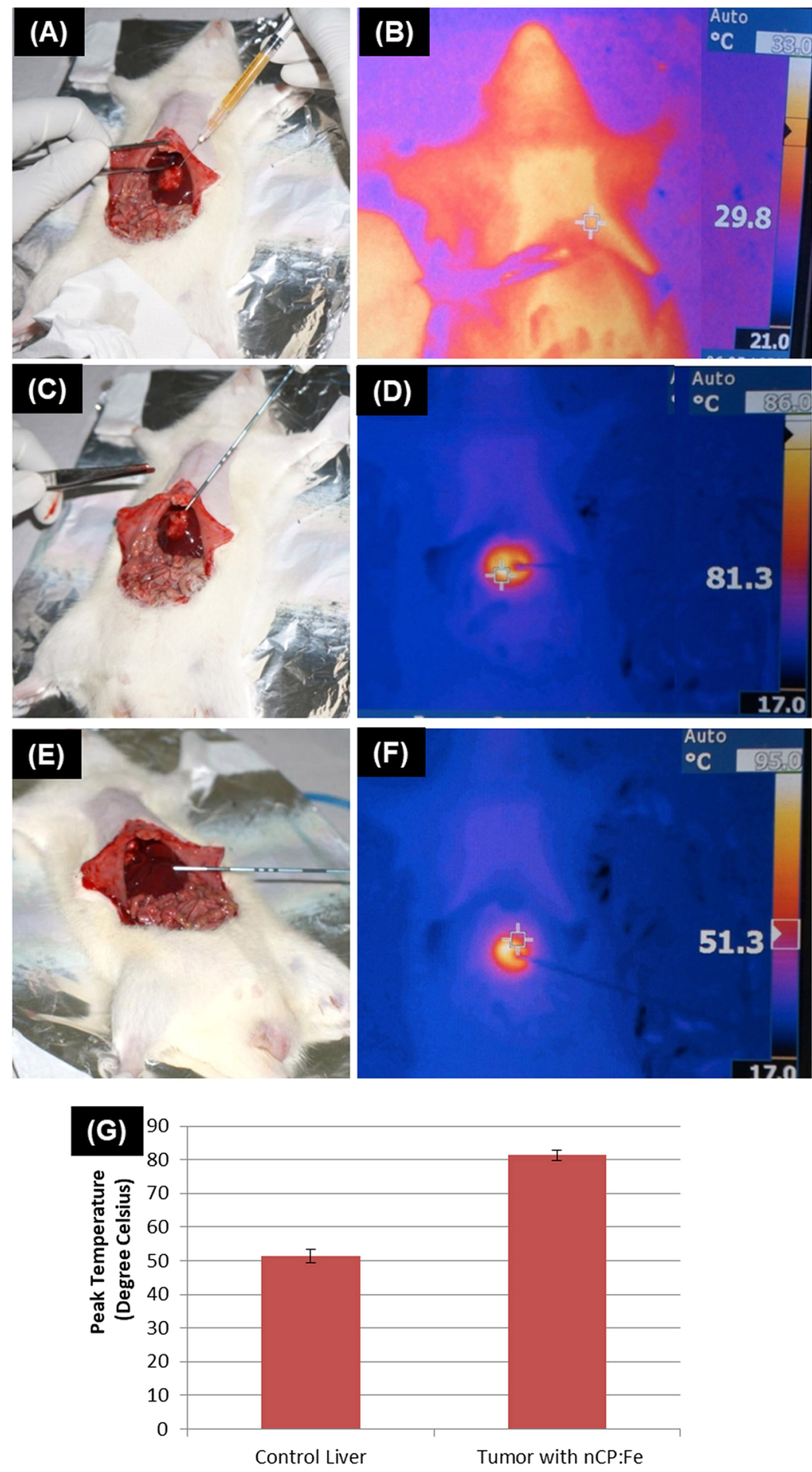

Figure 8. RFA of orthotopic liver tumor model and corresponding infrared images in comparison with healthy liver. (A) Photograph of sample being injected directly into tumor (B) IR image of the animal after sample injection (Peak Temp: $29.8 \pm 2{ }^{\circ} \mathrm{C}$ ) (C) Photograph of RFA procedure of sample injected liver tumor (D) Corresponding IR image after RFA (Peak Temp: $81.3 \pm 1.5^{\circ} \mathrm{C}$ ) (E) Photograph of RFA procedure of untreated healthy liver (F) IR image of the animal after RFA of untreated healthy liver region (Peak Temp: $51.3 \pm 2{ }^{\circ} \mathrm{C}$ ) (G) Graph showing the variation in temperature at the RF treatment point in nCP:Fe treated tumor and control healthy liver without nanoparticle.

In vitro Cell viability and Hemolysis analysis. Hemocompatibility analysis was carried out in human peripheral blood samples collected from healthy volunteers after the approval of the Institutional Human Ethics Committee, Amrita Institute of Medical Sciences and Research Centre, India. Informed consent was obtained 
from all subjects and all methods for humans were performed in accordance with the WHO guidelines for best practices in phlebotomy. In vitro cell viability was tested in PBMC isolated from human blood. PBMC was isolated using density gradient separation using Histopaque 1077, density $1.077 \mathrm{~g} / \mathrm{mL}$ (Sigma, USA). Cells were redispersed in RPMI complete medium containing $10 \% \mathrm{FBS}, 2 \mathrm{~mm}$ L-glutamine and $50 \mu \mathrm{M} \beta$ mercaptoethanol. $100 \mu \mathrm{L}$ of nCP:Fe of varying concentration from $20-500 \mu \mathrm{g} / \mathrm{mL}$, was taken in 96 well plates. $100 \mu \mathrm{L}$ of cells at a concentration of $10^{5}$ cells $/ \mathrm{mL}$ were seeded into the wells containing the samples. PBS was taken as the negative control and Triton X-100 as the positive control for the experiment. The cells were incubated with nCP:Fe for 48 hours at $37^{\circ} \mathrm{C} .100 \mu \mathrm{L}$ of $10 \%$ Alamar blue was added and incubated for 8 hours before the absorbance was measured at 570 and $600 \mathrm{~nm}$. For hemolysis analysis, whole blood was collected in $3.8 \%$ trisodium citrate anticoagulant (volume ratio of anticoagulant to blood $=1: 9$ ). $450 \mu \mathrm{L}$ of whole blood was treated with $50 \mu \mathrm{L}$ of nCP:Fe for 3 hours at $37^{\circ} \mathrm{C}$ under mild mixing. PBS and $1 \%$ Triton $\times 100$ was taken as the negative and positive control respectively. Blood was then centrifuged at $4000 \mathrm{rpm}$ for 15 minutes to collect plasma. Absorbance of plasma diluted with $0.01 \%$ sodium carbonate solution was measured spectrophotometrically (UV-1700, Shimadzu) at 380, 415 and $450 \mathrm{~nm}$. Amount of plasma hemoglobin was calculated as in Equation 1.

$$
\text { Amount of plasma hemoglobin }(m g / d L)=\frac{2 \times A_{415}-\left(A_{380}+A_{450}\right) \times 1000 \times \text { Dilution factor }}{(E \times 1.655)}
$$

where $\mathrm{A}_{415}, \mathrm{~A}_{380}, \mathrm{~A}_{450}$ are the absorbance values at 415,380 and $450 \mathrm{~nm}$. $\mathrm{A}_{415}$ is the soret band based absorption of hemoglobin. $\mathrm{A}_{380}$ and $\mathrm{A}_{450}$ are correction factors applied for uroporphyrin absorption. $\mathrm{E}$ is molar absorptivity value of oxyhemoglobin at $415 \mathrm{~nm}(\mathrm{E}=79.46) .1 .655$ is the correction factor applied due to the turbidity of plasma sample. \% Hemolysis was calculated as in Equation 2.

$$
\% \text { Hemolysis }=\frac{\text { Plasma } \mathrm{Hb} \text { value of sample }}{\text { Total } \mathrm{Hb} \text { value of blood }} \times 100
$$

where $\mathrm{Hb}$ is hemoglobin. For SEM imaging, RBC after particle treatment was incubated with $2.5 \%$ glutaraldehyde for 30 minutes. The cells were then washed twice in PBS before SEM imaging was carried out.

ROS generation analysis. DCFH-DA assay was used to measure the intracellular ROS after treatment of nCP:Fe with N1S1 hepatoma cells. Cells were seeded at a density of $2 \times 10^{5}$ cells per well in 6 well plates. nCP:Fe at a concentration of $250 \mu \mathrm{g} / \mathrm{mL}$ was added to cells. PBS and $30 \mu \mathrm{M} \mathrm{H}_{2} \mathrm{O}_{2}$ served as negative and positive controls respectively. The cells were incubated for 12 hours (4hours for positive control) and washed with PBS. $20 \mu \mathrm{M}$ of DCFH-DA in buffer was added to cells and incubated for 30 minutes at $37^{\circ} \mathrm{C}$. The cells are taken for flow cytometry (BDFACSAria II), intensity of fluorescence was detected with an excitation filter of $488 \mathrm{~nm}$ and band-pass emission filter of $530 \pm 15 \mathrm{~nm}$.

In vivo MRI and Bio-distribution analysis. All animal procedures were approved by the Institutional Animal Ethics Committee, Amrita Institute of Medical Sciences and Research Centre and the methods were done in accordance with guidelines of CPCSEA (Committee for the Purpose of Control and Supervision of Experiments on Animals, Govt. of India) guidelines. In vivo MRI was carried out in $7 \mathrm{~T}$ animal MRI station (BrukerBioSpec, USA). $10 \mathrm{mg} / \mathrm{kg}$ of nCP:Fe was injected intravenously into wistar rat. The animal was anesthetized in an induction chamber of isoflurane - oxygen mixture (5\% isoflurane). Rat was placed on the animal bed with a mask for providing maintenance dose of the anesthesia ( $2 \%$ isoflurane). A respiration pad and temperature probe with circulating warm water was used for continuous monitoring of respiration rate and body temperature. For $\mathrm{T}_{1}$ and $\mathrm{T} 2$ weighted $\mathrm{MRI}, \mathrm{a} \mathrm{T}_{1} \mathrm{FLASH}$ imaging sequence with $\mathrm{TR}=64 \mathrm{mS}, \mathrm{TE}=2.2 \mathrm{mS}, \mathrm{FA}=15^{\circ}$ and Turbo-RARE T2 imaging sequence with $\mathrm{TR}=2500 \mathrm{mS}, \mathrm{TE}=33 \mathrm{mS}, \mathrm{FA}=180^{\circ}$ was used respectively. For calculating T2 value of liver, T2 mapping sequence was carried out in axial liver sections. 3 different ROI was selected and the average T2 value was estimated. Biodistribution analysis was also carried out using the same parameters over a period of 96 hours. T1 and T2 weighted MRI of nCP:Fe injected intratumorally to subcutaneous tumor was also done using the above mentioned parameters. Organs were collected after euthanasia of animals by an overdose of the anesthetic (Xylazine: Ketamine =1:4). After washing each organ with PBS, it was weighed and homogenized. The homogenized tissue was dissolved in Tissue solvable (PerkinElmer) over a period of 24 hours and made up to $50 \mathrm{~mL}$ with $5 \%$ nitric acid for ICP-AES analysis to estimate $\mathrm{Fe}^{3+}$ content.

Prussian blue staining of RAW 264.7 macrophages. Cells were seeded to 24 well culture plates at a seeding density of 10,000 cells/ well and incubated overnight for cell attachment. The medium was then replaced with opti-MEM medium containing $100 \mathrm{ug} / \mathrm{mL}$ of $\mathrm{nCP}: \mathrm{Fe}$ and incubated for 12 hours. Medium was removed and cells were cultured in DMEM complete medium for 6 hours. Cells were then washed with PBS and fixed using $4 \%$ paraformaldehyde (Merck Co, India). Cells were then washed and treated with a mixture of $5 \%$ potassium ferrocyanide (Sigma-Aldrich Co, USA) and 5\% $\mathrm{HCl}$ (VeTEC, India) for 10 minutes. Cells were washed and stained with nuclear fast red (Merck- Millipore, USA) for $5 \mathrm{~min}$. Dehydration followed by mounting of coverslips was done. The cells were examined under optical microscope (Olympus, BX5).

In vitro RF response of Nanoparticles. RF response of the nanoparticles was measured in a custom made non-invasive 13.5 MHz RF instrument. Different concentrations of $\mathrm{nCP}: F e$ varying from $10-500 \mu \mathrm{g} / \mathrm{mL}$ was taken in a small glass petridish and $100 \mathrm{~W}$ RF power was applied for 1 minute. The temperature of the solution was measured before and after RF irradiation. RF response of nanoparticle treated N1-S1 hepatoma cells was also tested using the same non-invasive RF instrument. N1-S1 cells were seeded in 24 well plates at a seeding density of $2.5 \times 10^{4}$ cells/well. Different concentrations of nCP:Fe $(50-500 \mu \mathrm{g} / \mathrm{mL})$ was added to the wells. After incubation 
with the nanoparticles for 4 hours, the cells were irradiated with $100 \mathrm{~W}$ RF power for 5 minutes. 4 hours after RF treatment, media was changed. After 48 hours, cell viability analysis was carried out using Alamar blue assay. For estimation of RF response in tissue phantom, we chose goat liver tissue and invasive RF procedure was carried out using a cool tip RF ablation system (Cool-tip ${ }^{\mathrm{TM}} \mathrm{RF}$ ablation system, E series Covidien). The experiment was carried out in goat liver collected from 3 different animals, divided to two groups (i) PBS control group and (ii) $\mathrm{nCP}: \mathrm{Fe}$ group. $10 \mathrm{mg} / \mathrm{kg}$ of $\mathrm{nCP}: \mathrm{Fe}$ was injected to goat liver at a depth of $1 \mathrm{~cm}$. PBS was injected to control tissue. The RF probe was inserted (near to the point of sample injection) to a depth of $1 \mathrm{~cm}$ within the tissue and a power of $10 \mathrm{~W}$ was applied for 1 minute. After RF ablation, infrared (IR) image of the tissue was taken using IR imager (Fluke Ti200 Infrared camera) and diameter of the area of coagulation necrosis was measured.

In vivo Radiofrequency Ablation in Healthy Liver and Orthotopic Liver Tumor Model. Healthy adult wistar rats were divided into two groups (PBS control group and nCP:Fe group) containing 3 animals each. $10 \mathrm{mg} / \mathrm{kg}$ of nCP:Fe/PBS was injected intravenously to the rats. MRI was carried out before and after sample injection. Animals were then given an overdose of anesthesia. The abdomen region was opened and RF probe was inserted into the liver up to $1 \mathrm{~cm}$ depth. $10 \mathrm{~W}$ RF power was applied for a period of 1 minute using clinically used RFA system (Cool-tip ${ }^{\text {TM }}$ RF ablation system, E series Covidien). MRI of axial liver sections was carried out using TurboRARE T2 imaging sequence $\left(\mathrm{TR}=2500 \mathrm{mS}, \mathrm{TE}=33 \mathrm{mS}, \mathrm{FA}=180^{\circ}\right)$. The animals were then euthanized and liver was collected. $2 \mathrm{~mm}$ thick liver tissue was cut and live tissue staining was carried out using 2,3,5 Triphenyltetrazolium chloride (TTC) stain. The liver sections were incubated with $1 \%$ TTC stain at a temperature of $37^{\circ} \mathrm{C}$ for 30 minutes. Hematoxylin and Eosin staining was done to observe the extent of necrosis in tissue sections.

For development of orthotopic rat liver tumor models, 4-6 weeks old Sprague Dawley rats were injected with $1 \times 10^{6} \mathrm{~N} 1-\mathrm{S} 1$ rat hepatomacells (N1S1) under the capsule of left hepatic lobe. 10 days after injection tumor reached a size of $\sim 1.5 \mathrm{~cm}^{3} .300 \mu \mathrm{L}$ of $10 \mathrm{mg} / \mathrm{mL}$ sample was injected intratumorally to half of the tumor lobe. MRI was done to assess the particle distribution. RFA of the tumor was carried out at $10 \mathrm{~W}$ for 1 minute using clinically used RFA system (Cool-tip ${ }^{\mathrm{TM}}$ RF ablation system, E series Covidien). TTC staining of the liver was done to compare the viability of injected and control tumor region. The experiment was repeated in 3 orthotopic liver tumor rat models to obtain the average variation in necrosis area. Next, comparison of the increase in temperature of sample injected tumor region $v s$. healthy liver was carried out. Two groups (1. Orthotopic liver tumor rats 2. Healthy rat as control), each containing 3 animals were used for this study to obtain the average variation in tissue temperature. RFA (using Cool-tip ${ }^{\mathrm{TM}} \mathrm{RF}$ ablation system, E series Covidien) was done at $10 \mathrm{~W}$ for 1 minute. IR image (using Fluke Ti200 Infrared camera) was taken soon after RFA to compare the temperature increase in sample injected tumor vs control liver region.

\section{References}

1. Chan, W. C. \& Nie, S. Quantum dot bioconjugates for ultrasensitive nonisotopic detection. Science 281, 2016-2018 (1998).

2. Gao, X., Cui, Y., Levenson, R. M., Chung, L. W. \& Nie, S. In vivo cancer targeting and imaging with semiconductor quantum dots. Nat. Biotechnol. 22, 969-976 (2004).

3. Dubertret, B. et al. In vivo imaging of quantum dots encapsulated in phospholipid micelles. Science 298, 1759-1762 (2002).

4. Yong, K.-T. et al. Nanotoxicity assessment of quantum dots: from cellular to primate studies. Chem. Soc. Rev. 42, 1236-1250 (2013).

5. Derfus, A. M., Chan, W. C. \& Bhatia, S. N. Probing the cytotoxicity of semiconductor quantum dots. Nano Lett. 4, 11-18 (2004).

6. Torchilin, V. P. Recent advances with liposomes as pharmaceutical carriers. Nat. Rev. Drug Discovery 4, 145-160 (2005).

7. Allen, T. M. \& Cullis, P. R. Drug delivery systems: Entering the mainstream. Science 303, 1818-1822 (2004).

8. Lobovkina, T. et al. In vivo sustained release of siRNA from solid lipid nanoparticles. ACS Nano 5, 9977-9983 (2011).

9. Meng, H. et al. Engineered design of mesoporous silica nanoparticles to deliver doxorubicin and P-glycoprotein siRNA to overcome drug resistance in a cancer cell line. ACS nano 4, 4539-4550 (2010).

10. Desai, N. et al. Increased antitumor activity, intratumor paclitaxel concentrations, and endothelial cell transport of cremophor-free, albumin-bound paclitaxel, ABI-007, compared with cremophor-based paclitaxel. Clin. Cancer Res. 12, 1317-1324 (2006).

11. McCarthy, J. R., Perez, J. M., Brückner, C. \& Weissleder, R. Polymeric nanoparticle preparation that eradicates tumors. Nano lett. 5, 2552-2556 (2005).

12. Yu, M. K. et al. Drug-loaded superparamagnetic iron oxide nanoparticles for combined cancer imaging and therapy in vivo. Angew. Chem., Int. Ed. Engl. 47, 5362-5365 (2008).

13. Lanza, G. M. et al. Targeted antiproliferative drug delivery to vascular smooth muscle cells with a magnetic resonance imaging nanoparticle contrast agent. Circulation 106, 2842-2847 (2002).

14. McCarthy, J. R. \& Weissleder, R. Multifunctional magnetic nanoparticles for targeted imaging and therapy. Adv. Drug Delivery Rev. 60, 1241-1251 (2008).

15. Kim, D., Jeong, Y. Y. \& Jon, S. A drug-loaded aptamer-gold nanoparticle bioconjugate for combined CT imaging and therapy of prostate cancer. ACS nano 4, 3689-3696 (2010).

16. Huang, X., El-Sayed, I. H., Qian, W. \& El-Sayed, M. A. Cancer cell imaging and photothermal therapy in the near-infrared region by using gold nanorods. J. Am. Chem. Soc. 128, 2115-2120 (2006).

17. Loo, C., Lowery, A., Halas, N., West, J. \& Drezek, R. Immunotargeted nanoshells for integrated cancer imaging and therapy. Nano lett. 5, 709-711 (2005).

18. Lartigue, Ln et al. Cooperative organization in iron oxide multi-core nanoparticles potentiates their efficiency as heating mediators and MRI contrast agents. ACS nano 6, 10935-10949 (2012).

19. Kim, J. et al. Designed fabrication of a multifunctional polymer nanomedical platform for simultaneous cancer-targeted imaging and magnetically guided drug delivery. Adv. Mater. 20, 478-483 (2008).

20. Sanson, C. et al. Doxorubicin loaded magnetic polymersomes: theranostic nanocarriers for MR imaging and magnetochemotherapy. ACS nano 5, 1122-1140 (2011).

21. Lee, S. M. et al. Modular polymer-caged nanobins as a theranostic platform with enhanced magnetic resonance relaxivity and pHresponsive drug release. Angew. Chem., Int. Ed. Engl. 49, 9960-9964 (2010).

22. Pan, J., Liu, Y. \& Feng, S.-S. Multifunctional nanoparticles of biodegradable copolymer blend for cancer diagnosis and treatment. Nanomedicine (Lond). 5, 347-360 (2010).

23. Taratula, O. et al. A multifunctional theranostic platform based on phthalocyanine-loaded dendrimer for image-guided drug delivery and photodynamic therapy. Mol. Pharmaceutics 10, 3946-3958 (2013). 
24. Xu, X. et al. Bio-inspired supramolecular hybrid dendrimers self-assembled from low-generation peptide dendrons for highly efficient gene delivery and biological tracking. ACS nano 8, 9255-9264 (2014).

25. Lo, S.-T., Kumar, A., Hsieh, J.-T. \& Sun, X. Dendrimer nanoscaffolds for potential theranostics of prostate cancer with a focus on radiochemistry. Mol. Pharmaceutics 10, 793-812 (2013).

26. Saito, R. et al. Distribution of liposomes into brain and rat brain tumor models by convection-enhanced delivery monitored with magnetic resonance imaging. Cancer Res. 64, 2572-2579 (2004).

27. Ponce, A. M. et al. Magnetic resonance imaging of temperature-sensitive liposome release: drug dose painting and antitumor effects. J. Natl Cancer Inst. 99, 53-63 (2007)

28. Muthu, M. S., Kulkarni, S. A., Raju, A. \& Feng, S.-S. Theranostic liposomes of TPGS coating for targeted co-delivery of docetaxel and quantum dots. Biomaterials 33, 3494-3501 (2012).

29. Al-Jamal, W. T. \& Kostarelos, K. Liposomes: from a clinically established drug delivery system to a nanoparticle platform for theranostic nanomedicine. Acc. Chem. Res. 44, 1094-1104 (2011).

30. Lee, J. E. et al. Uniform mesoporous dye-doped silica nanoparticles decorated with multiple magnetite nanocrystals for simultaneous enhanced magnetic resonance imaging, fluorescence imaging, and drug delivery. J. Am. Chem. Soc. 132, 552-557 (2009).

31. Kim, J. et al. Multifunctional uniform nanoparticles composed of a magnetite nanocrystal core and a mesoporous silica shell for magnetic resonance and fluorescence imaging and for drug delivery. Angew. Chem., Int. Ed. Engl. 47, 8438-8441 (2008).

32. Kim, J., Piao, Y. \& Hyeon, T. Multifunctional nanostructured materials for multimodal imaging, and simultaneous imaging and therapy. Chem. Soc. Rev. 38, 372-390 (2009).

33. Merkle, E. M. et al. Effects of Superparamagnetic Iron Oxide on Radiofrequency-induced Temperature Distribution: In Vitro Measurements in Polyacrylamide Phantoms and in vivo Results in a Rabbit Liver Model. Radiology 212, 459-466 (1999).

34. Gu, L., Fang, R. H., Sailor, M. J. \& Park, J.-H. In vivo clearance and toxicity of monodisperse iron oxide nanocrystals. ACS nano 6, 4947-4954 (2012).

35. Mahmoudi, M., Laurent, S., Shokrgozar, M. A. \& Hosseinkhani, M. Toxicity evaluations of superparamagnetic iron oxide nanoparticles: cell "vision" versus physicochemical properties of nanoparticles. ACS nano 5, 7263-7276 (2011).

36. Apopa, P. L. et al. Iron oxide nanoparticles induce human microvascular endothelial cell permeability through reactive oxygen species production and microtubule remodeling. Part. Fibre Toxicol. 6, 1 (2009).

37. Ashokan, A. et al. Multifunctional calcium phosphate nano-contrast agent for combined nuclear, magnetic and near-infrared in vivo imaging. Biomaterials 34, 7143-7157 (2013).

38. Ashokan, A., Menon, D., Nair, S. \& Koyakutty, M. A molecular receptor targeted, hydroxyapatite nanocrystal based multi-modal contrast agent. Biomaterials 31, 2606-2616 (2010).

39. Ashokan, A. et al. Development and haematotoxicological evaluation of doped hydroxyapatite based multimodal nanocontrast agent for near-infrared, magnetic resonance and X-ray contrast imaging. Nanotoxicology 6, 652-666 (2012).

40. Barth, B. M. et al. Bioconjugation of calcium phosphosilicate composite nanoparticles for selective targeting of human breast and pancreatic cancers in vivo. ACS nano 4, 1279-1287 (2010).

41. Barth, B. M. et al. Targeted indocyanine-green-loaded calcium phosphosilicate nanoparticles for in vivo photodynamic therapy of leukemia. Acs Nano 5, 5325-5337 (2011).

42. Mi, P. et al. A pH-activatable nanoparticle with signal-amplification capabilities for non-invasive imaging of tumour malignancy. Nat. Nanotechnol. 11, 724-730 (2016).

43. Kester, M. et al. Calcium phosphate nanocomposite particles for in vitro imaging and encapsulated chemotherapeutic drug delivery to cancer cells. Nano lett. 8, 4116-4121 (2008).

44. Shin, T.-H. et al. T 1 and T 2 dual-mode MRI contrast agent for enhancing accuracy by engineered nanomaterials. ACS nano 8 , 3393-3401 (2014).

45. Carr, D. et al. Gadolinium-DTPA as a contrast agent in MRI: initial clinical experience in 20 patients. AJR, Am. J. Roentgenol. 143, 215-224 (1984).

46. Qin, J. et al. A High-Performance Magnetic Resonance Imaging T2 Contrast Agent. Adv. Mater. 19, 1874-1878 (2007).

47. Yang, H. et al. Targeted dual-contrast T 1 -and T 2 -weighted magnetic resonance imaging of tumors using multifunctional gadolinium-labeled superparamagnetic iron oxide nanoparticles. Biomaterials 32, 4584-4593 (2011).

48. Zhou, Z. et al. A synergistically enhanced T1-T2 dual-modal contrast agent. Adv. Mater. 24, 6223-6228 (2012).

49. Kunzli, B. M., Abitabile, P. \& Maurer, C. A. Radiofrequency ablation of liver tumors: Actual limitations and potential solutions in the future. World J. Hepatol. 3, 8-14 (2011).

50. Kettenbach, J. et al. Percutaneous saline-enhanced radiofrequency ablation of unresectable hepatic tumors: initial experience in 26 patients. AJR Am. J. Roentgenol. 180, 1537-1545 (2003)

51. Glazer, E. S. et al. Noninvasive radiofrequency field destruction of pancreatic adenocarcinoma xenografts treated with targeted gold nanoparticles. Clin. Cancer Res. 16, 5712-5721 (2010).

52. Gannon, C. J. et al. Carbon nanotube-enhanced thermal destruction of cancer cells in a noninvasive radiofrequency field. Cancer 110, 2654-2665 (2007).

53. Sasidharan, A. et al. Radiofrequency ablation of drug-resistant cancer cells using molecularly targeted carboxyl-functionalized biodegradable graphene. Adv. Healthcare Mater. 4, 679-684 (2015).

54. Hope, T. A. et al. Nephrogenic systemic fibrosis in patients with chronic kidney disease who received gadopentetate dimeglumine. Invest. Radiol. 44, 135-139 (2009).

55. Martins, M. A., Santos, C., Almeida, M. M. \& Costa, M. E. V. Hydroxyapatite micro-and nanoparticles: nucleation and growth mechanisms in the presence of citrate species. J. Colloid Interface Sci. 318, 210-216 (2008).

56. Mercado, D. F. et al. Paramagnetic iron-doped hydroxyapatite nanoparticles with improved metal sorption properties. A bioorganic substrates-mediated synthesis. ACS Appl. Mater. Interfaces 6, 3937-3946 (2014).

57. Tampieri, A. et al. Intrinsic magnetism and hyperthermia in bioactive Fe-doped hydroxyapatite. Acta Biomater. 8, 843-851 (2012).

58. Kolhatkar, A. G., Jamison, A. C., Litvinov, D., Willson, R. C. \& Lee, T. R. Tuning the magnetic properties of nanoparticles. Int. J. Mol. Sci. 14, 15977-16009 (2013).

59. Weissleder, R., Saini, S., Stark, D., Wittenberg, J. \& Ferrucci, J. Dual-contrast MR imaging of liver cancer in rats. AJR, Am. J. Roentgenol. 150, 561-566 (1988).

60. Simon, V. Iron Effect on Dielectric Properties of Calcium-Silica-Phosphate Glasses. Mod. Phys. Lett. B 16, 677-683 (2002).

61. Curley, S. A. Radiofrequency ablation of malignant liver tumors. The oncologist 6, 14-23 (2001).

62. Clasen, S. et al. MR-guided radiofrequency ablation in a 0.2 -T open MR system: Technical success and technique effectiveness in 100 liver tumors. J. Magn. Reson. Imaging 26, 1043-1052 (2007).

\section{Acknowledgements}

Authors thank, Department of Biotechnology (DBT), Govt of India for financial support under the projects "Translational Development of Protein nanomedicine and calcium phosphate nanocontrast agent" (BT/PK 7665/ NNT/28/658/2013) and support under BIG scheme for project titled "Nano-Biomineral Theranostic Agent for Image Guided RF Hyperthermia of Liver Tumor" (BIRAC/CCAMP0490/BIG-10/17). Authors thank Department of Science and Technology (DST) for financial support under the project "Theragnostics, Regenerative Medicine 
and Stem Cell Research using Cell-Targeted Nanomaterials" (SR/NM/NS-99/2009). Authors thank Mr. Sunil Kumar, Mr. Sajith, Ms. Sunitha for their technical assistance in animal experiments, Ms. Arya Raju for TEM and Mr. Sajin P Ravi for SEM analysis. Authors thank Sophisticated Test and Information Centre, CUSAT, Kerala for ICP-AES analysis.

\title{
Author Contributions
}

A.A. performed the experiments, analyzed data and prepared the manuscript. V.J. H. performed radiofrequency ablation experiments, G.S.G. carried out the in vitro and in vivo M.R.I. experiments, I.M.A. and R.B.J. participated in the synthesis of sterile nCP:Fe and its characterization, G.L.M. and B.S. helped in orthotopic tumor development, R.P. did the animal tumor induction procedures, A.K.K.U. supervised animal experiments, S.N. and M.K. supervised the work and corrected the manuscript.

\section{Additional Information}

Supplementary information accompanies this paper at https://doi.org/10.1038/s41598-017-14976-8.

Competing Interests: The authors declare that they have no competing interests.

Publisher's note: Springer Nature remains neutral with regard to jurisdictional claims in published maps and institutional affiliations.

\begin{abstract}
(c) (1) Open Access This article is licensed under a Creative Commons Attribution 4.0 International License, which permits use, sharing, adaptation, distribution and reproduction in any medium or format, as long as you give appropriate credit to the original author(s) and the source, provide a link to the Creative Commons license, and indicate if changes were made. The images or other third party material in this article are included in the article's Creative Commons license, unless indicated otherwise in a credit line to the material. If material is not included in the article's Creative Commons license and your intended use is not permitted by statutory regulation or exceeds the permitted use, you will need to obtain permission directly from the copyright holder. To view a copy of this license, visit http://creativecommons.org/licenses/by/4.0/.
\end{abstract}

(c) The Author(s) 2017 